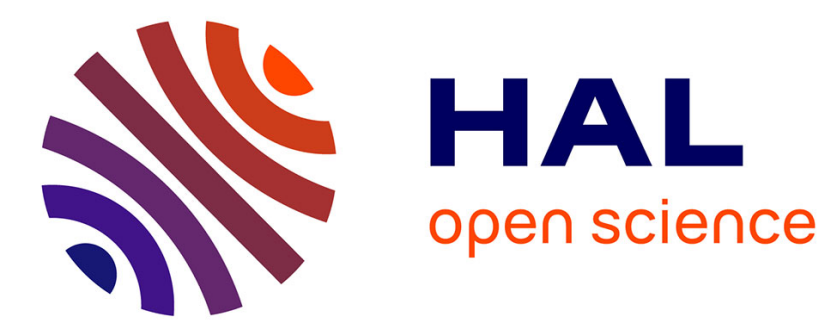

\title{
Cyclic negative feedback systems: what is the chance of oscillation?
}

\author{
Arnaud Tonnelier
}

\section{To cite this version:}

Arnaud Tonnelier. Cyclic negative feedback systems: what is the chance of oscillation?. Bulletin of Mathematical Biology, 2014, 76 (5), pp.1155-1193. 10.1007/s11538-014-9959-1 . hal-00919629

\section{HAL Id: hal-00919629 \\ https://hal.science/hal-00919629}

Submitted on 17 Dec 2013

HAL is a multi-disciplinary open access archive for the deposit and dissemination of scientific research documents, whether they are published or not. The documents may come from teaching and research institutions in France or abroad, or from public or private research centers.
L'archive ouverte pluridisciplinaire HAL, est destinée au dépôt et à la diffusion de documents scientifiques de niveau recherche, publiés ou non, émanant des établissements d'enseignement et de recherche français ou étrangers, des laboratoires publics ou privés. 


\title{
Cyclic negative feedback systems: what is the chance of oscillation?*
}

\author{
Arnaud Tonnelier \\ arnaud.tonnelier@inria.fr \\ INRIA-Grenoble, \\ 655 avenue de l'Europe, Montbonnot \\ 38334 Saint Ismier, France \\ Tel.: +33-4-76615572
}

\begin{abstract}
Many biological oscillators have a cyclic structure consisting of negative feedback loops. In this paper, we analyze the impact that the addition of a positive or a negative self-feedback loop has on the oscillatory behaviour of the three negative feedback oscillators proposed by Tsai et al (Science 231:126-129, 2008) where, in contast with numerous oscillator models, the interactions between elements occur via the modulation of the degradation rates. Through analytical and computational studies we show that an additional self-feedback affects the dynamical behaviour. In the high cooperativity limit, i.e. for large Hill coefficients, we derive exact analytical conditions for oscillations and show that the relative location between the dissociation constants of the Hill functions and the ratio of kinetic parameters determines the possibility of oscillatory activities. We compute analytically the probability of oscillations for the three models and show that the smallest domain of periodic behaviour is obtained for the negative-plus-negative feedback system whereas the additional positive self-feedback loop does not modify significantly the chance to oscillate. We numerically investigate to what extent the properties obtained in the sharp situation applied in the smooth case. Results suggest that a switchlike coupling behaviour, a time-scale separation and a repressilator-type architecture with an even number of elements facilitate the emergence of sustained oscillations in biological systems. An additional positive selffeedback loop produces robustness and adaptability whereas an additional negative self-feedback loop reduces the chance to oscillate.
\end{abstract}

Keywords : Oscillation - Feedback - Limit cycle - Repressilator - Helmholtz decomposition

\footnotetext{
*To the memory of José Manuel Zaldívar Comenges.
} 


\section{Introduction}

Connected networks arising in systems biology show a wide variety of dynamical behaviours, oscillations being a recurrent motif. Oscillations frequently occur in the regulation of biological systems and play a fundamental role in numerous physiological processes as the hormone secretion (Walker et al. (2012)), the cardiac electrical activity (Keener and Sneyd (1998)), the circadian rhythm (Goldbeter (2002)) or in pharmacodynamics (Dokoumetzidis et al. (2001)), to mention a few. Therefore, an important topic in mathematical biology has been the study of necessary conditions for a system to show and maintain oscillations in a fluctuating environment such as the interior of a cell (Weber et al. (2011); Mincheva (2011); Tsai et al. (2008); Ferrell et al. (2011) and references therein). Determining the oscillatory regimes of a system described by a set of coupled nonlinear differential equations is well recognized as a very difficult problem and finding conditions on a system's parameters for which a limit cycle exists is revealed to be a major challenge. This issue has been extensively tackled through numerical simulations and analytical approaches. Algebraic criteria for biochemical reaction networks described with mass action law kinetics have been derived (Mincheva and Roussel (2007); Boulier et al. (2007)). Generalized mass action kinetics and nonmass action kinetics, such as Michaelis-Menten and Hill kinetics, have been studied in (Mincheva (2011)) and (Mincheva and Cracium (2008)), respectively. These approaches are based on the existence of a supercritical Andronov-Hopf bifurcation (Kuznetsov (2004)) and provide a restricted set of parameters for which small oscillations exist (Tyson (1975)). However the absence of Hopf bifurcations cannot preclude the existence of a limit cycle that could appear through another bifurcation. Necessary conditions for oscillations based on a generalized version of the Bendixon-Dulac criterion have been derived and applied to polynomial vector fields (Weber et al. (2011)).

Sustained oscillations most often result from the interaction of different components forming complex networks. It has been suggested that the dynamical properties of the system could be understood in terms of network connectivity considering the so-called interaction graph (Thomas (1981); Kaufman et al. (2007); Mincheva (2011); Domijan and Pécou (2012); Purcell et al. (2010)). A hallmark of robust oscillations is the existence of inhibitory feedback loops despite the fact that the existence of a negative feedback circuit is not necessary (Richard and Comet (2011)) ${ }^{1}$. Negative feedbacks are frequently embedded in a cyclic architecture (Fraser and Tiwari (1974); Hastings et al. (1977); Smith (1987); Mallet-Paret and Smith (1990); Goldbeter (1991); Elkhader (1992); Müller et al. (2006)) that is believed to be the underlying circuit responsible for the emergence of oscillations in networks. The cyclic nature of interactions appears in the description of cascades of enzimatic reactions coupled with gene transcription (see references in (Hastings et al. (1977))), in cellular control systems or in neural systems where the term 'ring' is used (see various exemples

\footnotetext{
${ }^{1}$ The local interaction graph of an oscillatory system does not have necessarily a negative circuit. However the global interaction graph must have a negative circuit of length at least two.
} 
and references in section 4 of (Mallet-Paret and Smith (1990))). The repressilator, a well-known synthetic genetic regulatory network, uses a cyclic repression architecture (Elowitz and Leibler (2000); Müller et al. (2006)). For this class of models, many results can be found in the literature. A proof of the existence of large amplitude oscillations for a three dimensional cyclic negative feedback system with one nonlinearity dates back to Tyson (1975). The proof is based on the construction of an invariant torus and the use of a fixed point theorem. Hastings (1977) has shown a similar result replacing the sigmoidal response by a step function and has provided results on the unicity and stability of the limit cycle. A general nonlinearity has been tackled by Smith (1986). The extension to an arbitrary dimension and to a general class of nonlinearities has been performed by Hastings et al. (1977). Moreover it has been observed (Fraser and Tiwari (1974)) and proved (Smith (1987)) that a qualitative difference occurs in the dynamics of negative cyclic systems between an odd number and an even number of nodes in the cycle. A significant insight has been accomplished by Mallet-Paret and Smith (1990): they have shown that monotone cyclic feedback systems can be embedded in $R^{2}$ and, therefore, the possible dynamics of the network are severely constrained. Common characteristics of global attractors for generic cyclic feedback systems have been described by Gedeon and Mischaikow (1995) and Gedeon (1998).

Even though negative feedback loops are enough to generate oscillations (Goldbeter (1991); Griffith (1968); Elowitz and Leibler (2000); Hirata et al. (2002); Tsai et al. (2008)), many biological oscillators have also positive feedback loops (Harris and Levine (2005); Hornung and Barkai (2008); Angeli et al. (2004); Guantes and Poyatos (2006)) raising the question of the functional role of these extra loops. It has been shown that the regulation of cell cycle is based on a combination of positive and negative feedback loops (Goldbeter (2002)). Genetic oscillators are frequently modeled by a two-component system of repressoractivator type (see for instance Guantes and Poyatos (2006)). There is an extensive literature on the coupling of positive and negative feedback loops generating p53 oscillations (Ciliberto et al. (2005); Harris and Levine (2005)). Several explanations have been proposed to justify the existence of positive loops in biological systems. A conjecture by Thomas (Thomas (1981)) demonstrated in (Snoussi (1998); Gouzé (1998); Plahte et al. (1995); Cinquin and Demongeot (2002)) states that the presence of a positive feedback loop is a necessary condition for biological systems being able to possess multiple steady states. In particular a bistable behavior can be obtained inducing a switch-like response (Angeli et al. (2004); Cherry and Adler (2000)). Tsai et al. (2008) argued that the existence of a positive feedback loop makes oscillatory systems easy to tune and more robust (see also (Stricker et al. (2008))). This is an important characteristic that living systems should possess when exposed to a changing environment (Stricker et al. (2008)). Moreover it is believed that positive control elements might improve the reliability of the oscillations and induce high noise-resistance (Elowitz and Leibler (2000)). Positive feedback loops allow the stabilization of active steady states (López-Caamal et al. (2013)) whereas positive-plus-negative feedback loops are thought to be involved in the balance 
between noise buffering and signaling sensitivity (Hornung and Barkai (2008)). The study of the network architecture and its relation with the dynamical behaviour has attracted a lot of attention but the geometrical properties of the vector field generating the flow of the system have been poorly explored. A first attempt in this direction has been carried out by Demongeot et al. (2007a,b). They shown that a close link exists between sustained oscillations and the existence of a potential-Hamiltonian decomposition of the vector field. For Liénard polynomial system, the Hamiltonian part of the decomposition provides an accurate approximation of the limit cycle. However the link with the interaction graph of the system and the applicability of the method to a dimension greater than two have not been addressed. There is a need to fill the gap between oscillations, interaction graph and vector-field properties.

In this paper, we study the cyclic inhibitory feedback systems considered by Tsai et al. (2008) where the symmetry of the cycle can be broken by an additional negative or positive self-feedback loop. In Sect. 2 we present the models. In Sect. 3.1 we discuss the nature of the interactions and in Sect. 3.2 we exhibit a vector-field decomposition of the 3D system. Fixed points are studied in Sect. 3.3 and a qualitative description of oscillations in terms of slow-fast dynamics is provided (Sect. 3.4). In the idealized case of step-like coupling, we derive exact conditions for the existence of oscillations and we describe analytically the ranges of model parameter where stable limit cycles can be found (Sect. 3.5). Numerical simulations in the smooth-coupling case are performed in Sect. 3.6. We generalize some results to large cyclic repression systems in Sect. 3.7 and we conclude by a discussion (Sect. 4).

\section{Models}

The dynamics that controls the synthesis and degradation of molecules is an elaborate process supported by a complex circuitry forming positive and negative feedback loops. All the feedbacks are not active at the same time and it is thought that only small size circuits are involved in the modulation of the molecule concentration at a given period of its life (Harris and Levine (2005)). In this context, simple oscillators have been used as minimal models to unravel the complexity of network dynamics (Ferrell et al. (2011); Purcell et al. (2010)). Among them cyclic three-component oscillators have attracted a lot of attention (Hastings (1977); Goldbeter (1991); Ferrell et al. (2011)). In this paper the oscillator models formulated in (Tsai et al. (2008)) are considered (see also (Angeli et al. (2004)) and (Ferrell et al. (2011))): each oscillator is modeled by a dynamical system of three variables where the temporal evolution is driven by a linear term plus a Hill-type coupling term. The first oscillator model, referred to as the negative feedback-only oscillator (the No-oscillator in the sequel) is described by a set of three coupled non linear differential equations

$$
\frac{d A}{d t}=k_{1}(1-A)-k_{2} A S_{1}(C),
$$




$$
\begin{aligned}
& \frac{d B}{d t}=k_{3}(1-B)-k_{4} B S_{2}(A), \\
& \frac{d C}{d t}=k_{5}(1-C)-k_{6} C S_{3}(B) .
\end{aligned}
$$

Variables, $A, B$ and $C$, represent a concentration of a molecule or a chemical species; a gene, a protein or a metabolite. Parameters $\left(k_{i}\right)_{i=1, \ldots, 6}>0$ are rate constants and $\left(S_{i}\right)_{i=1,2,3}$ are the Hill functions

$$
S_{i}(x)=\frac{x^{n_{i}}}{K_{i}^{n_{i}}+x^{n_{i}}},
$$

where $n_{i}>0$ are Hill coefficients and $K_{i}>0$ are the median effective concentration values of the Hill functions (i.e. the half-maximal concentrations). Parameters $K_{i}$ are also referred to as the microscopic dissociation constants or saturation constants, or when Hill functions have a sharp transition, as thresholds or switching values. Without cooperative binding, i.e. for $n_{i}=1$, the interactions follow the Michaelis-Menten kinetic model, $K_{i}$ being the Michaelis constant.

Two other oscillators have been introduced: the negative-plus-negative oscillator (NN-oscillator in the sequel) and the positive-plus-negative oscillator (PNoscillator in the sequel). These oscillators have an additional self-feedback loop on the A-component of the No-oscillator. For the NN-oscillator, equation (1) becomes

$$
\frac{d A}{d t}=k_{1}(1-A)-k_{2} A S_{1}(C)-k_{7} A S_{4}(A),
$$

and for the PN-oscillator a positive feedback is introduced

$$
\frac{d A}{d t}=k_{1}(1-A)-k_{2} A S_{1}(C)+k_{7}(1-A) S_{4}(A) .
$$

where $S_{4}$ is given by (2). The No-oscillator belongs to the class of repressilatortype models (Elowitz and Leibler (2000)) that are characterized by the cyclic inhibitory connection of elements (proteins that cyclically repress the synthesis of another). The presence of a positive self-feedback loop for the PN-oscillator is a characteristic feature of amplified negative feedback oscillators (Purcell et al. (2010)) where one species amplifies its own production.

The system is endowed with properties frequently encountered in regulatory systems. The internal dynamics of each molecule is described by a linear term that combines a constant (normalized) production with a degradation term (or dilution) using a constant relaxation time $\tau_{i}=1 / k_{i}$. The couplings between components are monotone (a positive influence remains positive at all concentrations ${ }^{2}$, and vice versa) and are described by standard Hill's equations multiplied by the concentration of the associated molecule. It is worth noting that the coupling term can be rewritten as a modulation of the degradation rates

${ }^{2}$ Except for the PN-oscillator (see Sect. 3.1) 
unlike many regulatory models where the coupling acts on the synthesis term (we will discuss this point latter, section 3.5.2). A schematic view of the oscillators is provided in Fig. 1.

Due to the Bendixson's and Dulac's criteria, it is well known that the two com-

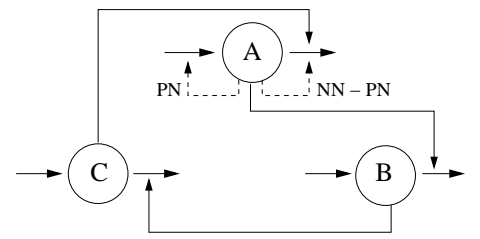

Figure 1: Schematic view of the oscillator models. The additionnal loops of the negative-plus-negative oscillator (labelled NN) and positive-plus-negative oscillator (labelled PN) are represented in dotted lines.

ponents version of the No-oscillator and NN-oscillator are not able to generate oscillations and a minimal oscillator with negative interactions only has at least three components. The three-node architecture proposed for the No-oscillator model appears as a recurrent network motif in many different independent biological contexts (Pigolotti et al. (2007)). It has been widely used as a generic and realistic minimal model responsible for the oscillatory behaviour observed in complex networks ( Goldbeter (1991); Ferrell et al. (2011); Boulier et al. (2007); Pigolotti et al. (2007)). The ring architecture made of successive negative interactions is a common motif in regulatory networks or signaling systems (Angeli et al. (2004); Pigolotti et al. (2007)).

Recently, a similar model of repressilator-type has been studied by Buse et al. $(2009,2010)$. It has been shown that if the negative feedback is sufficiently strong and if the sigmoidal interaction is sufficiently stiff then the system oscillates. For the models studied here, it has been numerically observed by Tsai et al. (2008) that the three oscillator models exhibit oscillations and that oscillatory regimes are more easily obtained in the positive-plus-negative type oscillator whereas oscillations are limited by the negative self-feedback loop.

\section{Results}

\subsection{The nature of interactions}

The positive or negative nature of a loop is determined by the sign of the partial derivative of the functions involved in the loop and is commonly represented by an interaction graph. The sign of the $(i, j)$ component of the Jacobian matrix (see Appendix A), i.e. $\partial f_{i} / \partial x_{j}$, defines the nature of the connection from $j$ to $i$ and the product of the signs of the components defining the loop gives the nature of the feedback loop. In Tsai et al. (2008) another convention seems to be used since the sign of the scalar term describing the feedback in the equations 
determines the nature of the interaction. For the oscillators studied here the two definitions coincide except for the nature of the self-feedback loop of the PN-oscillator. The positive interaction term $(1-A) S_{4}(A)$ in the PN-oscillator equation has a positive derivative on $\left(0, A^{*}\right)$ and a negative derivative on $\left(A^{*}, 1\right)$ where $A^{*}$ is the unique solution of $x^{n_{4}+1}+\left(1+n_{4}\right) K_{4}^{n_{4}} x-n_{4} K_{4}^{n_{4}}=0$. Adding the linear term $k_{1}(1-A)$ the nature of the self-interaction remains variable and can become negative in particular when $k_{1}$ is sufficiently large. In the limit of large $n_{4}$ values, it is easy to show that the maximum of the derivative of the interaction term $(1-A) S_{4}(A)$ is reached for $A=K_{4}$ (when $K_{4}<1$ ) and the feedback is non-negative if and only if

$$
\frac{k_{1}}{k_{7}}<\frac{n_{4}}{4}\left(\frac{1}{K_{4}}-1\right)-\frac{1}{2}
$$

to a leading order in $1 / n_{4}$. To sum up, the interactions between molecules induce a negative coupling. In addition, each molecule has a negative self-feedback loop describing the degradation but it is common to ignore it in the interaction graph (Fig. 1). The additional loop of the NN-oscillator does not change the nature of the self-feedback loop whereas for the PN-oscillator the nature of the loop becomes variable: it is negative when the degradation dominates and positive when amplification dominates. By abuse of language the PN-oscillator is referred to as an amplified negative feedback oscillator.

The oscillator models previously introduced can be generalized and written in an abstract form as

$$
\frac{d x_{i}}{d t}=f_{i}\left(x_{i}, x_{i-1}\right), \quad i=1,2, \ldots, n
$$

where we set $x_{0}=x_{n}$ with $n$ the number of species involved in the cycle. For the negative feedback-only model we have:

$$
f_{i}\left(x_{i}, x_{i-1}\right)=k_{2 i-1}\left(1-x_{i}\right)-k_{2 i} x_{i} S_{i}\left(x_{i-1}\right), \quad i=1,2, \ldots, n .
$$

For the NN-model and PN-model an additional loop, that depends on $x_{1}$ only, is included in the definition of $f_{1}$ (see eq. (3) and eq. (4), respectively). It can be checked easely that $D=] 0,1{ }^{n}$ is positively invariant. Moreover we have

$$
\frac{\partial f_{i}\left(x_{i}, x_{i-1}\right)}{\partial x_{i-1}}=-k_{2 i} x_{i} S_{i}^{\prime}\left(x_{i-1}\right)<0, \quad \forall x \in D \text { and } 1 \leq i \leq n
$$

and therefore the different oscillators belong to the class of monotone cyclic feedback systems (Mallet-Paret and Smith (1990)). It is worth noting that the models considered here do not satisfy the properties of the systems presented in (Hastings et al. (1977)) and in (Smith (1987)) and therefore previous theorems on limit cycle existence do not apply here.

If we define $u_{i}$ such that $x_{i}=e^{u_{i}}$, then the cyclic feedback system can be rewritten in a more standard decoupled form as

$$
\frac{d u_{i}}{d t}=a_{i}\left(u_{i}\right)-b_{i}\left(u_{i-1}\right), \quad i=1,2, \ldots, n
$$

where $a_{i}(x)=k_{2 i-1}\left(e^{-x}-1\right)$ and $b_{i}(x)=k_{2 i} S_{i}\left(e^{x}\right)$. 


\subsection{Helmholtz decomposition, energy dissipation and di- vergence}

Oscillations in biological systems have been widely analyzed via the associated interaction graph but the geometrical properties of the system have been poorly addressed. One can expect that the structure of the vector field provides an intuitive understanding of the dynamics and gives some key elements to figure out the appearance of stable periodicity.

Let us define $S_{k}^{[1]}(x)=\int_{0}^{x} S_{k}(u) d u$ and $S_{k}^{[2]}(x)=\int_{0}^{x} S_{k}^{[1]}(u) d u$. Both integrals can be analytically expressed with the generalized hypergeometric functions ${ }_{p} F_{q}\left(a_{1}, \ldots, a_{p} ; b_{1}, \ldots, b_{q} ; x\right)$ as

$$
\begin{aligned}
& S_{k}^{[1]}(x)=x\left(1-{ }_{2} F_{1}\left(1,1 / n_{k} ; 1+1 / n_{k} ;-\left(x / K_{k}\right)^{n_{k}}\right)\right), \\
& S_{k}^{[2]}(x)=x\left(1-{ }_{3} F_{2}\left(1,1 / n_{k}, 2 / n_{k} ; 1+2 / n_{k}, 1+1 / n_{k} ;-\left(x / K_{k}\right)^{n_{k}}\right)\right) .
\end{aligned}
$$

Let $F_{i}$ be the vector field of oscillator $i$ where $i \in\{\mathrm{No}, \mathrm{NN}, \mathrm{PN}\}$ stands for one of the three different oscillators. The oscillators share a similar Helmholtz decomposition of their vector field

$$
F_{i}=-\nabla \phi_{i}+\nabla \times G
$$

where $\phi_{i}$ is a scalar potential defining the conservative part of the vector field. The vector potential $G$ is identical for all oscillators and is given by

$$
G(A, B, C)=\left(\begin{array}{c}
k_{6} C S_{3}^{[1]}(B) \\
k_{2} A S_{1}^{[1]}(C) \\
k_{4} B S_{2}^{[1]}(A)
\end{array}\right) .
$$

The scalar potential $\phi_{i}$ can be decomposed as $\phi_{i}=\phi+\tilde{\phi}_{i}$ where $\phi$ is a potential common to all oscillators given by

$$
\begin{aligned}
\phi(A, B, C)= & \frac{k_{1}}{2}(1-A)^{2}+k_{4} S_{2}^{[2]}(A)+\frac{k_{3}}{2}(1-B)^{2}+k_{6} S_{3}^{[2]}(B)+\ldots \\
& +\frac{k_{5}}{2}(1-C)^{2}+k_{2} S_{1}^{[2]}(C) .
\end{aligned}
$$

$\tilde{\phi}_{i}$ is an oscillator-dependent potential induced by the self-feedback loop and is given by

$$
\begin{aligned}
\tilde{\phi}_{N o}(A) & =0 \\
\tilde{\phi}_{N N}(A) & =k_{7} A S_{4}^{[1]}(A)-k_{7} S_{4}^{[2]}(A), \\
\tilde{\phi}_{P N}(A) & =k_{7}(A-1) S_{4}^{[1]}(A)-k_{7} S_{4}^{[2]}(A) .
\end{aligned}
$$

The vector field of each oscillator is the sum of a curl-free vector field, defined by $\phi_{i}$, and a divergence-free vector field, defined by $G$. The divergence-free part defines the circulation density (source free) of the oscillators and remains 
the same in the three versions of the oscillators. The curl-free part defines the source density and reflects the difference between each oscillator induced by the additional self-feedback. The modification of the source density term can be measured directly by the divergence of the vector field. Physically the divergence measures to which extent the flow generated by the vector field behaves as a source or a sink. The divergence describes the rate of change of an infinitesimal state space volume $V(t)$ following the flow defined by $F$ and we have $\operatorname{div}(F(x))=$ $\dot{V}(t) / V(t)$. In conservative systems there is no change of the total energy and therefore the state space volume is constant and $\operatorname{div} F=0$. In non-conservative systems, if $\operatorname{div} F>0$ the volume $V(t)$ increases and the vector field flow behaves like a source, whereas if $\operatorname{div} F<0$ then $V(t)$ decreases and the vector field flow behaves as a sink. We have

$$
\operatorname{div} F_{i}=-\Delta \phi_{i},
$$

and we calculate for the No-oscillator

$$
\operatorname{div} F_{N o}=-k_{1}-k_{3}-k_{5}-k_{2} S_{1}(C)-k_{4} S_{2}(A)-k_{6} S_{3}(B)
$$

which is negative. For the NN-oscillator, we get

$$
\operatorname{div} F_{N N}=\operatorname{div} F_{N o}-k_{7} S_{4}(A)-k_{7} A S_{4}^{\prime}(A)
$$

which is still negative. For the PN-oscillator, we obtain

$$
\operatorname{div} F_{P N}=\operatorname{div} F_{N o}-k_{7} S_{4}(A)+k_{7}(1-A) S_{4}^{\prime}(A)
$$

which has $k_{7}(1-A) S_{4}^{\prime}(A)$ as a positive term in the divergence traducing the existence of a positive self-feedback. It is easy to show that the sum of the two terms adding $\operatorname{div} F_{N o}$ in the right hand side of (11) is positive when $A \in\left(0, A^{*}\right)$ where $0<A^{*}<1$ is the unique solution of $n_{4} K_{4}^{n_{4}}-\left(n_{4}+1\right) K_{4}^{n_{4}} x-x^{n_{4}}=0$. It is worth noting that the existence of an Helmhotz decomposition is not insured by the Helmholtz-Hodge theorem which applies for a decaying vector field that vanishes at infinity. Here, the system lies on a bounded space (the cube $[0,1]^{3}$ ) where the decomposition is not unique. It is easy to check that $\phi=k_{1} A+k_{2} B+$ $k_{3} C$ and $G=0.5\left(k_{2} C-k_{3} B, k_{3} A-k_{1} C, k_{1} B-k_{2} A\right)^{t}$ satisfy $\nabla \phi=\nabla \times G$.

\subsection{Steady states}

Without interactions between molecules, i.e. $k_{2}=k_{4}=k_{6}=k_{7}=0$, the oscillators have $(A, B, C)=(1,1,1)$ as a globally attractive steady state. The negative coupling modifies the nature of the resting state and qualitatively the new fixed points result from a balance between an attraction towards $(1,1,1)$ driven by the internal dynamics and an attraction towards $(0,0,0)$ generated by the inhibitory connection. If a static balance can not be reached, a dynamical state induced by the negative interactions emerges with possibly the birth of oscillations.

Steady states are points in the state space where the curl-free component equals 
the divergence-free component of the vector field. For oscillator $i$, the steady state $X_{0}=\left(A_{0}, B_{0}, C_{0}\right)$ satisfies

$$
\nabla \phi_{i}\left(X_{0}\right)=\nabla \times G\left(X_{0}\right) .
$$

For the No-oscillator we obtain

$$
\begin{aligned}
& A_{0}=\left(1+r_{2,1} S_{1}\left(C_{0}\right)\right)^{-1}, \\
& B_{0}=\left(1+r_{4,3} S_{2}\left(A_{0}\right)\right)^{-1}, \\
& C_{0}=\left(1+r_{6,5} S_{3}\left(B_{0}\right)\right)^{-1},
\end{aligned}
$$

where $r_{p, q}$ is the ratio of rate constants $k_{p} / k_{q}$. The functions $f_{k}: x \rightarrow 1 /(1+$ $\left.r_{k} S_{k}(x)\right)$ ) (where $r_{k}=r_{2 k, 2 k-1}$ ) are decreasing and thus

$$
A_{0}=f_{1}\left(f_{3}\left(f_{2}\left(A_{0}\right)\right)\right)
$$

has at most one solution. Since $f_{1}\left(f_{3}\left(f_{2}(0)\right)\right)>0$ and $f_{1}\left(f_{3}\left(f_{2}(x)\right)\right)-x<0$ for sufficiently large $x$, equation (12) has exactly one solution. The stability is given by the eigenvalues of the jacobian matrix. For the NN-oscillator, using similar arguments one can show that there is exactly one steady state. However for the PN-oscillator several steady states can coexist as shown in Fig. 2.

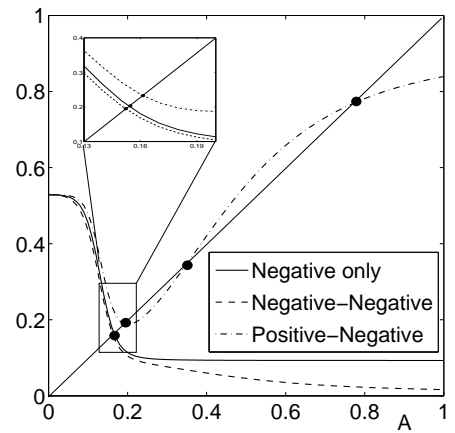

Figure 2: Determination of the A-component of the steady states as the crossing point between the curves and the straight line $y=A$, for the different oscillators. For the negative feedback-only oscillator and the negative-plus-negative oscillator there exists one fixed point. For the positive-plus-negative oscillator, several steady states can coexist. The inset shows a zoom on the corresponding region. Parameters are $r_{2,1}=r_{4,3}=r_{6,5}=10, r_{7,1}=100, K_{1}=K_{2}=K_{4}=0.2$, $K_{3}=0.25$ and $n_{1}=n_{2}=n_{3}=n_{4}=3$.

It is worth noting that the No-oscillator is invariant under the circulating permutation

$$
\begin{aligned}
& k_{1} \quad \rightarrow k_{3} \quad \rightarrow k_{5} \quad \rightarrow k_{1}, \\
& k_{2} \rightarrow k_{4} \quad \rightarrow k_{6} \quad \rightarrow k_{2} \text {, } \\
& K_{1} \rightarrow K_{2} \rightarrow K_{3} \rightarrow K_{1} \text {, } \\
& n_{1} \quad \rightarrow n_{2} \quad \rightarrow n_{3} \quad \rightarrow n_{1} .
\end{aligned}
$$


The symmetry is broken by the additional self-feedback loop and thus, in comparison with the No-oscillator, the possible oscillator birth or oscillator death obtained for the $\mathrm{NN}$-oscillator or the $\mathrm{PN}$-oscillator is induced by symmetry breaking.

\subsection{The fast-slow repressilator}

A qualitative understanding of the emergence of oscillations is provided by a fast-slow timescale analysis of the negative feedback oscillators. Let us consider the No-oscillator where we assume that the rate constants $k_{1}, k_{3}$ and $k_{5}$ are small, i.e. $k_{1}, k_{3}, k_{5} \sim \epsilon$ where $\epsilon \ll 1$. If the sigmoidal interactions are sufficiently sharp, thus starting from an underthreshold initial condition (i.e. each sigmoidal function $S_{i}$ is inactivated), the system evolves following the slow dynamics

$$
\begin{aligned}
& \frac{d A}{d t}=k_{1}(1-A), \\
& \frac{d B}{d t}=k_{3}(1-B), \\
& \frac{d C}{d t}=k_{5}(1-C),
\end{aligned}
$$

that continues as long as the concentrations of the different molecules are below their associated thresholds, i.e. $A<K_{2}, B<K_{3}$ and $C<K_{1}$. When one species reaches its threshold, the corresponding interaction is activated. Depending on the relative location of $K_{1}, K_{2}, K_{3}$ and the relative strength of the rate constants $k_{1}, k_{3}$ and $k_{5}$, different oscillatory patterns can be obtained. Let us assume that $C$ reaches its threshold $K_{1}$ first. At that time, noted $t_{0}$, the concentration of molecule $A$ follows the fast dynamic

$$
\frac{d A}{d t}=-k_{2} A S_{1}(C)
$$

and quickly tends towards 0 . We enter a regime where $A(t) \ll 1$ and $B, C$ slowly tend towards 1 . Let $t_{1}$ be the time at which $B\left(t_{1}\right)=K_{3}$. At that time, $C$ tends towards 0 following a fast dynamics whereas $A$ enters a slow recovery process towards 1 while $B$ continues to increase towards 1 . At a time noted $t_{2}$, $A$ reaches its threshold and subsequently $B$ is reset to 0 whereas $C$ starts its recovery process that will define a time $t_{3}$ such that $C\left(t_{3}\right)=K_{1}$. These different regimes repeat indefinitely giving rise to an oscillatory activity characterized by the successive resetting of variables $A, B$ and $C$ (fast trajectory towards 0 ) following by recovery processes towards 1 (see Fig. 3). It should be noted that the fast-slow repressilator presented here has a fast repressor dynamics in contrast with many synthetic oscillators where the activation is fast and repression is slow (Purcell et al. (2010)).

From the previous discussion, it is easy to show that a necessary and sufficient condition to have a periodic switch between the fast and the slow regimes is 


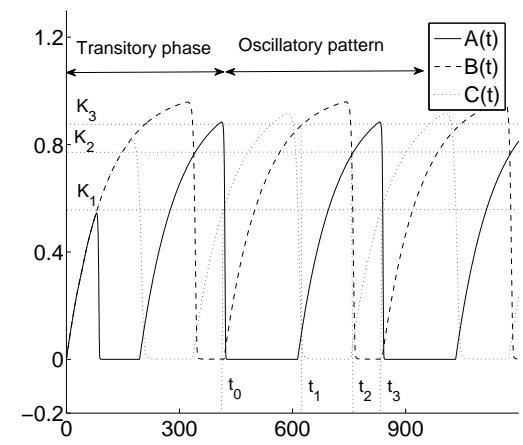

Figure 3: Oscillatory activity of the fast-slow negative feedback-only oscillator as a function of time. Parameters are $k_{1}=k_{3}=k_{5}=0.01, k_{2}=30, k_{4}=10$ and $k_{6}=10$. The threshold values are $K_{1}=0.6, K_{2}=0.8$ and $K_{3}=0.9$. The Hill coefficients are $n_{1}=n_{2}=n_{3}=100$.

$K_{i}<1, i=1,2,3$. The analysis is valid in the limit of a sharp sigmoid, i.e. $n_{i} \gg 1$, and we will show in the next section that this condition prevents the occurence of fixed points that would lead to oscillator death.

\subsection{The high cooperativity limit}

The Hill functions describe the cooperative dynamics of macromolecules. Interactions of Hill type are modeled by sigmoidal functions where the slope of the curve near the median effective concentration is governed by the Hill coefficient. When the Hill coefficient is large $\left(n_{i} \gg 1\right)$, a situation that we refer to as the high cooperativity limit, the transition around $K_{i}$ is sharp and the sigmoid behaves as an Heaviside-step function generating a switch-like interaction. The functions $S_{i}$, eq. (2), become

$$
S_{i}(x)=\Theta\left(x-K_{i}\right)
$$

where $\Theta$ is the Heaviside step function and parameter $K_{i}$ plays the role of a threshold. The idealization of nonlinear functions by an Heaviside step function to address oscillations dates back to McKean (1970) and Hastings (1977). It has been long-past observed that many biological interactions exhibit a switchlike behaviour and threshold-dominated systems have attracted a widespread interest (see references in (Mestl et al. (1995a))). The resulting piecewise linear systems have been proposed as a modelling framework in biology allowing efficient simulations while being analytically tractable. Piecewise linear systems have been introduced for the study of regulatory networks (Glass and Kaufman (1973); Mestl et al. (1995a); Gouzé and Sari (2003)) and have been successfully applied to the qualitative simulation of genetic networks (de Jong et al. (2003)). 
Earlier results on the possible oscillatory regimes have been obtained by Glass and Pasternack (1978a) and greatly extended by Snoussi (1989), Farcot and Gouzé (2010) and Lu and Edwards (2010).

For the piecewise linear version of the oscillators studied here, it is possible to derive analytical conditions for the existence of oscillations. Let us define the following critical values

$$
K_{1}^{*}=\frac{k_{5}}{k_{5}+k_{6}}, \quad K_{2}^{*}=\frac{k_{1}}{k_{1}+k_{2}}, \quad K_{3}^{*}=\frac{k_{3}}{k_{3}+k_{4}} .
$$

For the NN-oscillator, we define the two additional critical values

$$
K_{4, a}^{*}=\frac{k_{1}}{k_{1}+k_{2}+k_{7}}, \quad K_{4, b}^{*}=\frac{k_{1}}{k_{1}+k_{7}}
$$

and for the PN-oscillator, we define

$$
K_{4}^{*}=\frac{k_{1}+k_{7}}{k_{1}+k_{2}+k_{7}} .
$$

Each critical value is the ratio of the rate constants of the reaction kinetics with and without interactions. We find that the conditions for the existence of an oscillatory regime are determined by the relative location of $K_{i}, i=1,2,3,4$ with the associated critical values $K_{i}^{*}$ and with unity (see appendix B.2). More precisely, for the No-oscillator, oscillations exist when

$$
\begin{aligned}
& K_{1}^{*}<K_{1}<1, \\
& K_{2}^{*}<K_{2}<1, \\
& K_{3}^{*}<K_{3}<1 .
\end{aligned}
$$

For the NN-oscillator, the existence of oscillations is given by the following non-intersecting sets

$$
\begin{aligned}
& K_{1}^{*}<K_{1}<1, \quad K_{1}^{*}<K_{1}<1, \\
& K_{2}^{*}<K_{2}<1, \text { or } K_{4, a}^{*}<K_{2}<K_{4, b}^{*} \text {, } \\
& K_{3}^{*}<K_{3}<1, \text { or } K_{3}^{*}<K_{3}<1 \text {, } \\
& K_{4}>1, \quad 0<K_{4}<K_{4, b}^{*} .
\end{aligned}
$$

For the PN-oscillator, we find

$$
\begin{aligned}
& K_{1}^{*}<K_{1}<1, \\
& K_{2}^{*}<K_{2}<1, \\
& K_{3}^{*}<K_{3}<1, \\
& K_{4}>K_{4}^{*},
\end{aligned}
$$

The projection of the sets (13), (14) and (15) on the $\left(K_{2}, K_{4}\right)$ parameter plane is shown in Fig. 4. An example of a limit cycle together with the corresponding oscillatory pattern is plotted in Fig. 5. When one of the inequalities defining the sets (13), (14) and (15) is violated, a stable fixed point appears (see appendix B.1) leading to the so-called oscillator death. When $K_{4}>1$, the additional self-feedback loop of the $\mathrm{NN}$ and PN-oscillator is inactive and the conditions for the existence of oscillations are identical for all oscillators, i.e. $K_{i}^{*}<K_{i}<$ $1, i=1,2,3$. 


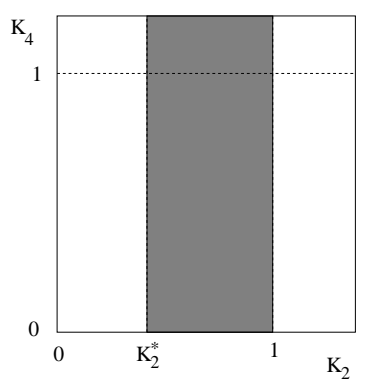

$A$

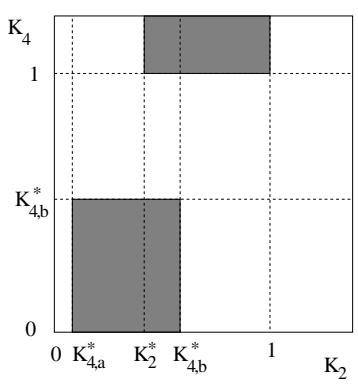

$B$

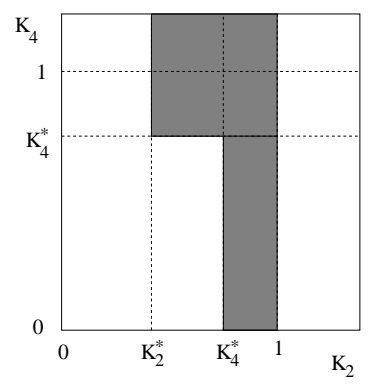

C

Figure 4: Locus of existence of stable oscillations in the $\left(K_{2}, K_{4}\right)$ parameter space (shaded grey) for (A) the negative feedback-only oscillator, (B) the negative-plus-negative oscillator and $(\mathrm{C})$ the positive-plus-negative oscillator. Parameters, $K_{i}, i=1,3$, satisfied $K_{i}^{*}<K_{i}<1$.

\subsubsection{Remarks on oscillatory activities}

The oscillatory patterns that we obtained are expected to be limit cycles, i.e. periodic trajectories. The main reason is based on the results of Mallet-Paret and Smith (1990) on smooth monotone feedback systems and its possible generalization to discontinuous systems: without stable fixed points, the trajectories of the system approach as $t \rightarrow \infty$, either a limit cycle, or a set consisting of equilibra together with homoclinic and hetereclinic orbits. In particular, chaotic solutions do not occur. Homoclinic or heteroclinic orbits do not exist in the studied systems. The proof is trivial for regular fixed points and we assume that the possible singular fixed points do not support these connecting orbits. Numerically we only observed limit cycles or fixed points.

We have, strictly speaking, only derived sufficient conditions for the existence of oscillatory patterns. These conditions are necessary if we assume that the existence of a stable fixed point precludes the existence of stable limit cycles, i.e. bistability between a fixed point and a limit cycle is ruled out. Many negative cyclic feedback systems share this property (Tyson (1975); Hastings (1977); Hastings et al. (1977); Smith (1987); Mallet-Paret and Smith (1990); Müller et al. (2006), and other references therein) that holds for systems where oscillations occur through a supercritical Hopf bifurcation, in contrast with the subcritical Hopf bifurcation that may support bistability. Thus we have rigorously derived only a subset of the total oscillatory domain but, numerically, we did not observed bistability.

Additional complications in the analysis are generated by the nature of the flow on the switching surfaces where singular solutions may exist (Glass and Pasternack (1978a,b); Snoussi and Thomas (1993); Mestl et al. (1995a)). A rigorous definition of these states can be found in the Filippov textbook (Filippov (1988)). Another way to study dynamics on singular domain is the singular 


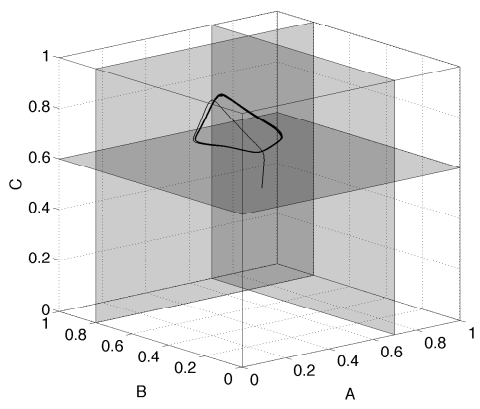

$A$

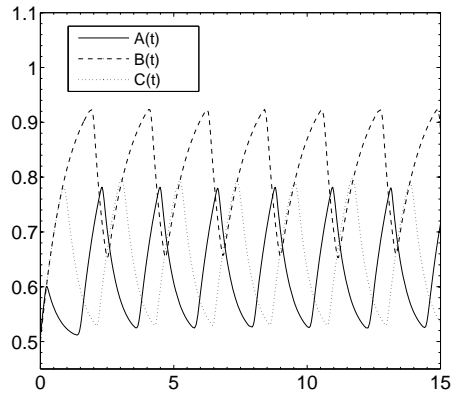

$B$

Figure 5: Oscillations in the piecewise linear negative feedback-only oscillator. A) Limit cycle in the phase plane. The phase plane is partitioned into rectangular regions delimited by the switching planes $A=K_{2}, B=K_{3}$ and $C=K_{1}$. B) The corresponding trajectories of the three variables of the model. Parameters are $k_{i}=1, i=1, \ldots, 6$ and $K_{1}=0.6, K_{2}=0.7, K_{3}=0.8$. The initial condition is $(A(0), B(0), C(0))=(0.5,0.5,0.5)$.

perturbation method of Plahte and Kjoglum (2005) and Ironi et al. (779-794). These approaches have been recently revisited by Machina et al. (2013) and reformulated in the complementarity systems framework by Acary et al. (2013) . Due to the particular nature of the systems studied here, there are no oscillatory trajectories with a sliding part but steady states may lie on a switching surface. Such steady states are the so-called singular steady states in contrast with the regular steady states commonly encountered in smooth dynamical systems. The study of the existence and stability of steady states done in appendix B.1 encompasses these two cases.

It is worth noting that (i) the limit cycles do not occur through the destabilization of a fixed point undergoing an Andronov-Hopf bifurcation and therefore we are not limited here to small size limit cycles. (ii) Multistability between two stable fixed points exists only for the PN-oscillator: one fixed point satisfies $A_{s s}=K_{4}^{*}$ and the other $A_{s s}=K_{2}^{*}$. This is in agreement with the result stating that positive loops are responsible for multistability (Snoussi (1998)). (iii) When a regular fixed point exists, it is stable and only singular fixed points can be unstable. In particular, the singular fixed point $\left(K_{2}, K_{3}, K_{1}\right)$ may exist for the three different oscillators and is always unstable. For the NN- and PNoscillator an additional singular fixed point may occur on the switching surface $A=K_{4}$ and can be stable for the NN-oscillator whereas it is always unstable for the PN-oscillator. 


\subsubsection{Links with previous works}

The analytical treatment of the piecewise linear oscillators uses a partition of the phase space into orthants defined by the thresholds. A common representation of the dynamics uses the so-called state transition diagram of the system (Glass and Pasternack $(1978 \mathrm{a}, \mathrm{b}))$. The state transition diagram is a directed graph that can be assigned to piecewise linear systems due to the constant direction of the flow across the common boundary of neighboring orthants. The nodes represent orthants, the arrows denote the direction of the flow between orthants and a boolean variable is associated to each orthant. For instance 010 corresponds to the configuration $A<K_{2}, B>K_{3}$ and $C<K_{1}$. The state transition diagram does not depend on the precise values of the parameters but rather on the location of the so-called focal points. In Fig. 6 we plot the state transition diagram of the No-oscillator for $k_{i}=1, i=1, \ldots, 6$ and $K_{i}=2 / 3, i=1,2,3$ showing the existence of a so-called logical cycle. Since there is no branching point the logical cycle is a cyclic attractor of the system. A conjecture of

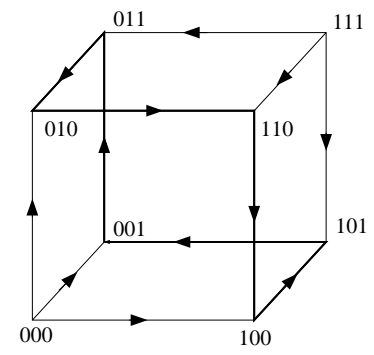

Figure 6: State transition diagram for the negative feedback-only oscillator where $K_{i}^{*}<K_{i}<1, i=1,2,3$. The cyclic attractor is represented by heavy edges.

Glass and Pasternack (1978a) (conjecture 2) stated that the existence of a cyclic attractor in the state transition diagram implies the existence of a stable limit cycle in the associated piecewise-linear differential system. This conjecture has been refined and shown in (Glass and Pasternack (1978b)) for piecewise linear systems with identical decay rates: if the state transition diagram supports a cyclic attractor then the corresponding differential system tends towards a limit cycle or asymptotically approaches the origin, the origin being the singular steady state where each variable equals to its threshold value. A similar theorem has been previously derived in (Hastings (1977)) for a three dimensional system. Recent outcomes that generalize previous results have been obtained by Farcot and Gouzé $(2009,2010)$ for non uniform decay rates and multiple interaction loops.

To make a comparison with our analysis it is convenient to rewrite the equations in the following form

$$
\frac{d x_{i}}{d t}=\alpha_{i}(X)-\gamma_{i}(X) x_{i}, \quad i=1,2, \ldots, n
$$


where $X$ is the boolean vector $X=\left(\Theta\left(x_{1}-K_{2}\right), \Theta\left(x_{2}-K_{3}\right) \ldots, \Theta\left(x_{n}-K_{1}\right)\right)$, $\alpha_{i}(X)$ denotes the production rate and $\gamma_{i}(X)$ is the relative decay rate. Formulation (16) is commonly used to describe the dynamics of regulatory genetic networks (Glass and Pasternack (1978b); Mestl et al. (1995a,b)). However only one threshold per variable is used and thus the $\mathrm{PN}$ - and NN-oscillators cannot be recasted in this framework. Moreover common assumptions are to consider identical decay rates and to use the production term to describe the interactions, in contrast with the No-oscillator studied here where the coupling is defined through the degradation rate

$$
\gamma_{i}(X)=k_{2 i-1}+k_{2 i} X_{i-1},
$$

(we define $X_{0}=X_{n}$ ) and the production rate is assumed to be constant

$$
\alpha_{i}(X)=k_{2 i-1} .
$$

Oscillations are therefore induced by the modulation of the decay rates and not by the time evolution of production rates. Mathematically, the equations present piecewise constant decay rates unlike the vast majority of previous work that uses constant rates and piecewise constant production terms. Moreover another significative difference with previous work on periodic orbits (Glass and Pasternack (1978b); Farcot and Gouzé (2009)) is the interaction structure of the model: each variable inhibits the production of the next variable that differ from systems where each variable activates the next variable except one (noted $x_{n}$ ) which inhibits $x_{1}$.

The cyclic attractor of the transition graph is closely related to the existence of an absorbing torus-like region for the smooth system (Buse et al. (2010)) which is conected with the partition of the state space into several regions where the sign of the flow remains constant. The signature of the dynamics is a pattern similar to the one obtained here (Buse et al. (2009)): (110) $\rightarrow(100) \rightarrow(101) \rightarrow$ $(001) \rightarrow(011) \rightarrow(010)$ which is remiscent to the functioning of the ring oscillator (an odd number of not gate in series forming a chain) in electronics.

Finally, a closely related study in term of methodology has been performed by Mahaffy (1980) in the field of neurosciences. Sufficient conditions for oscillations in neural networks are derived from stable fixed points analysis. Similarly to our approach, the author uses a step function to idealize the nonlineraties, considers inhibitory connections and focuses on circular architectures. Analytical expressions on network structure are obtained for a network to generate and sustained oscillations (including chaotic solutions).

\subsubsection{Probability of oscillations}

A Monte Carlo approach has been used by Tsai et al. (2008) to explore in the parameter space the domain of existence of stable limit cycles. They generated random parameter sets for the three different oscillators and determined with the numerical integration of the equations whether oscillatory regimes are observed. One of the goals of this paper is to provide an analytical understanding of the results numerically obtained by the authors (Fig. 4 in (Tsai et al. 
$(2008)))$. On the other hand, determining the probability of oscillations has a twofold interest. First it allows to tackle the issue of robustness of oscillations and, secondly, it provides a quantitative measure of the percentage of oscillatory cells.

In the limit $n_{i} \rightarrow \infty$ we have obtained in the previous section the domain of existence of oscillatory activities. Thus we will derive analytically the probability of oscillations for the different oscillator models.

Negative feedback-only oscillator. Let $P_{N o}$ be the probability of the No-oscillator oscillating. We have

$$
P_{N o}=\prod_{i=1,2,3} P\left(K_{i}^{*}<K_{i}<1\right)
$$

or equivalently

$$
P_{N o}=\prod_{i=1,2,3}\left(1-P\left(K_{i}>1\right)-P\left(K_{i}<K_{i}^{*}\right)\right)
$$

Let us assume that the thresholds, $K_{i}, i=1,2,3$, are random variables that follow continuous uniform distributions on $\left[0, \bar{K}_{i}\right]$ and we note $K_{i} \sim U\left(0, \bar{K}_{i}\right)$ where $\left(\bar{K}_{i}\right)$ are positive constants that we assume greater than 1 for simplicity. We also assume that the rate constants $k_{j}, j=1, \ldots, 6$ satisfy $k_{i} \sim U\left(0, \bar{k}_{i}\right)$.

We have

$$
P\left(K_{i}>1\right)=1-\frac{1}{\bar{K}_{i}} .
$$

Using $K_{1}^{*}=k_{5} /\left(k_{5}+k_{6}\right)$, we obtain

$$
P\left(K_{1}<K_{1}^{*}\right)=\frac{1}{\bar{K}_{1} \bar{k}_{5} \bar{k}_{6}} \int_{0}^{\bar{k}_{6}} \int_{0}^{\bar{k}_{5}} \frac{x}{x+y} d x d y .
$$

We define

$$
F(x)=\frac{\ln (1+x)}{x}
$$

and we calculate

$$
P\left(K_{1}<K_{1}^{*}\right)=\frac{1}{2 \bar{K}_{1}}\left(F\left(\frac{\bar{k}_{6}}{\bar{k}_{5}}\right)-F\left(\frac{\bar{k}_{5}}{\bar{k}_{6}}\right)+1\right)
$$

and similar equations hold for the expressions of $P\left(K_{2}<K_{2}^{*}\right)$ and $P\left(K_{3}<K_{3}^{*}\right)$. Let $(\mathrm{H})$ be the following assumption: the thresholds $\left(K_{i}\right), i=1,2,3$, the self rate constants $\left(k_{2 i-1}\right)$ and the coupling rate constants $\left(k_{2 i}\right)$ have identical and independent uniform distributions. We note

$$
(H)\left\{\begin{array}{l}
K_{i} \sim U(0, K) \\
k_{2 i-1} \sim U(0, k) \\
k_{2 i} \sim U\left(0, k_{c}\right)
\end{array}\right.
$$


where $K \geq 1, k>0$ and $k_{c}>0$ where the subscript 'c' stands for 'coupling'. The probability of oscillations for the No-oscillator is given by

$$
P_{N o}=\frac{1}{8 K^{3}}\left(1+F\left(r_{c}\right)-F\left(\frac{1}{r_{c}}\right)\right)^{3}
$$

where $r_{c}$ is the ratio of the maximal value of the self rate constant over the maximal value of the coupling rate constant, i.e. $r_{c}=k / k_{c}$. Probability (22) is maximum when $r_{c} \rightarrow 0$, and, for small $r_{c}$ values, we derive the following asymptotic expansion

$$
P_{N o}=\frac{1}{K^{3}}\left(1-\frac{3}{4} r_{c}+\frac{3}{2} r_{c} \ln r_{c}\right)+r_{c} \epsilon\left(r_{c}\right),
$$

where $\epsilon\left(r_{c}\right) \rightarrow 0$ as $r_{c} \rightarrow 0$. For parameters used in (Tsai et al. (2008)) (see appendix C) we find $P_{N o}=0.01446$. Approximation (23) gives $P_{N o}=0.01443$ and if we keep only the first term of the expansion we find $P_{N_{o}}=0.01562$.

In (Tsai et al. (2008)) the value of $k_{5}$ has been fixed to 1 and therefore $K_{1}^{*}=$ $\left(1+k_{6}\right)^{-1}$. Expression (18) becomes:

$$
P\left(K_{1}<K_{1}^{*}\right)=\frac{1}{\bar{K}_{1} \bar{k}_{6}} \int_{0}^{\bar{k}_{6}} \frac{d y}{1+y}
$$

which gives

$$
P\left(K_{1}<K_{1}^{*}\right)=\frac{1}{\bar{K}_{1}} F\left(\bar{k}_{6}\right)
$$

and we find $P\left(K_{1}<K_{1}^{*}\right)=1.7210^{-3}$ (instead of $P\left(K_{1}<K_{1}^{*}\right)=6.3910^{-3}$ when $\left.k_{5} \sim U(0, k)\right)$ that yields $P_{N o}=0.0147$.

Negative-plus-negative oscillator. For the NN-oscillator, we assume that the parameters of the self-feedback loop satisfy $k_{7} \sim U\left(0, \bar{k}_{7}\right)$ and $K_{4} \sim U\left(0, \bar{K}_{4}\right)$. From (14) the probability of oscillations for the NN-oscillator is given by

$$
\begin{aligned}
P_{N N}= & P_{N o} P\left(K_{4}>1\right) \\
& +P\left(K_{1}^{*}<K_{1}<1\right) P\left(K_{4, a}^{*}<K_{2}<K_{4, b}^{*}\right) P\left(K_{3}^{*}<K_{3}<1\right) P\left(K_{4}<K_{4, b}^{*}\right) .
\end{aligned}
$$

Both probabilities $P\left(K_{1}^{*}<K_{1}<1\right)$ and $P\left(K_{3}^{*}<K_{3}<1\right)$ have been previously calculated analytically (see (17) and (20)). We have $P\left(K_{4}>1\right)=1-1 / K_{4}$ and both probabilities, $P\left(K_{4}<K_{4, b}^{*}\right)$ and $P\left(K_{2}<K_{4, b}^{*}\right)$, are given by (20) substituting $\bar{k}_{7} / \bar{k}_{1}$ to $\bar{k}_{6} / \bar{k}_{5}$ and using $\bar{K}_{4}$ and $\bar{K}_{2}$, respectively, instead of $\bar{K}_{1}$. To complete the analytical expression of $P_{N N}$, it remains to calculate $P\left(K_{2}<\right.$ $K_{4, a}^{*}$ ). We get

$$
P\left(K_{2}<K_{4, a}^{*}\right)=\frac{1}{\bar{K}_{2} \bar{k}_{1} \bar{k}_{2} \bar{k}_{7}} \int_{0}^{\bar{k}_{1}} \int_{0}^{\bar{k}_{2}} \int_{0}^{\bar{k}_{7}} \frac{x}{x+y+z} d x d y d z
$$


which has an analytical expression given in appendix B.3 (eq. (32)).

Assuming that $(\mathrm{H})$ holds, we obtain

$$
\begin{aligned}
P_{N N}= & P_{N o}\left(1-\frac{1}{K}\right) \\
& +\frac{1}{16 K^{4}}\left(1-F\left(\frac{1}{r_{c}}\right)+F\left(r_{c}\right)\right)^{2}\left(1+F\left(\frac{1}{r_{s}}\right)-F\left(r_{s}\right)\right) \\
& \times\left(1+F\left(\frac{1}{r_{s}}\right)-F\left(r_{s}\right)-2 K I_{N N}\right)
\end{aligned}
$$

where $I_{N N}$ is the integral in the right-hand side of $(24)$ and $r_{s}=k / \bar{k}_{7}$ measures the strength of the self-feedback loop. Taking the limit $r_{c} \rightarrow 0$ we have $I_{N N} \rightarrow 0$ that yields

$$
P_{N N}=\frac{1}{K^{3}}\left(1-\frac{1}{K}\right)+\frac{1}{4 K^{4}}\left(1+F\left(\frac{1}{r_{s}}\right)-F\left(r_{s}\right)\right)^{2}+\epsilon\left(r_{c}\right)
$$

To compare with the No-oscillator, we calculate the zero-order expansion of the ratio of probabilities

$$
\frac{P_{N N}}{P_{N o}}=1-\frac{1}{K}+\frac{1}{4 K}\left(1+F\left(\frac{1}{r_{s}}\right)-F\left(r_{s}\right)\right)^{2}
$$

for small $r_{c}$ values. The ratio is maximum when $r_{s} \rightarrow \infty$ and we have $P_{N N} / P_{N o} \rightarrow$ 1 that corresponds to the case where $k_{7} \rightarrow 0$, i.e. the self-feedback is inactivated and the two oscillators coincide. The minimum is reached for $r_{s} \rightarrow 0$ (strong self-feedback) and we have $P_{N N} / P_{N o} \rightarrow 1-1 / K$. Expression (25) has been derived assuming $r_{c} / r_{s} \rightarrow 0$ and therefore we only capture the limiting regime $r_{c} \rightarrow 0, r_{s} \rightarrow 0$ and $r_{c} / r_{s} \rightarrow 0$, i.e. the coupling strength remains greater (of at least one order) than the self-feedback strength.

For a strong self-feedback and a strong coupling, both of the same order, we consider $r_{c}=r_{s}=r \ll 1$, and, from the complete expression of $P_{N N}$, we calculate

$$
P_{N N}=\frac{1}{K^{3}}\left(1-\frac{1}{K}\right)+\epsilon(r)
$$

which coincides with the asymptotic development $(25)$ when $r_{s} \rightarrow 0$.

For parameters used in (Tsai et al. (2008)) (see appendix (C)), we find $P_{N N}=$ 0.0109 using the exact analytical expression. Asymptotic expansion (25) gives $P_{N N}=0.0118$. In the so-called 'strong positive feedback' case, i.e. $r_{c}=r_{s}=$ 0.01 , we find $P_{N N}=0.0108$ and approximation (26) gives 0.0117 .

Positive-plus-negative oscillator. As for the NN-oscillator, we take $k_{7} \sim U\left(0, \bar{k}_{7}\right)$ and $K_{4} \sim U\left(0, \bar{K}_{4}\right)$. From (15) the probability of oscillations for the PNoscillator is given by

$$
\begin{aligned}
P_{P N}= & P_{N o} P\left(K_{4}>K_{4}^{*}\right) \\
& +P\left(K_{1}^{*}<K_{1}<1\right) P\left(K_{4}^{*}<K_{2}<1\right) P\left(K_{3}^{*}<K_{3}<1\right) P\left(K_{4}<K_{4}^{*}\right) .
\end{aligned}
$$


All the probabilities occuring in the expression of $P_{P N}$ have been calculated analytically hereinabove except $P\left(K_{4}<K_{4}^{*}\right)$ and $P\left(K_{2}<K_{4}^{*}\right)$. We have

$$
P\left(K_{4}<K_{4}^{*}\right)=\frac{1}{\bar{K}_{4} \bar{k}_{1} \bar{k}_{2} \bar{k}_{7}} \int_{0}^{\bar{k}_{1}} \int_{0}^{\bar{k}_{2}} \int_{0}^{\bar{k}_{7}} \frac{x+z}{x+y+z} d x d y d z
$$

that can be rewritten as

$$
P\left(K_{4}<K_{4}^{*}\right)=\frac{1}{\bar{K}_{4}}-I_{P N}
$$

where

$$
I_{P N}=\frac{1}{\bar{K}_{4} \bar{k}_{1} \bar{k}_{2} \bar{k}_{7}} \int_{0}^{\bar{k}_{1}} \int_{0}^{\bar{k}_{2}} \int_{0}^{\bar{k}_{7}} \frac{y}{x+y+z} d x d y d z
$$

has been previously calculated analytically (from eq. (24), switching $k_{2}$ and $k_{1}$ ). A similar expression holds for $P\left(K_{2}<K_{4}^{*}\right)$.

Assuming that $(\mathrm{H})$ holds, we calculate

$$
P_{P N}=P_{N o}\left(1-\frac{1}{K}+I_{P N}\right)+\frac{1}{4 K^{2}}\left(1+F\left(r_{c}\right)-F\left(\frac{1}{r_{c}}\right)\right)^{2} I_{P N}\left(\frac{1}{K}-I_{P N}\right) .
$$

Taking the limit $r_{c} \rightarrow 0$ we get $I_{P N} \rightarrow 1 / K$ and we obtain

$$
P_{P N}=\frac{1}{K^{3}}+\epsilon\left(r_{c}\right)
$$

that yields $P_{N P} / P_{N o} \rightarrow 1$. The limit does not depend on $r_{s}$ unlike the NNoscillator. For a strong negative coupling and a strong self-feedback, we note $r=r_{c}=r_{s} \rightarrow 0$. We find $I_{P N} \rightarrow 1 /(2 K)$ and we calculate

$$
P_{P N}=\frac{1}{K^{3}}\left(1-\frac{1}{4 K}\right)+\epsilon(r)
$$

that is lower than the limit (27) obtained as $r_{c} \rightarrow 0$ and $r_{s}$ fixed.

For $K=4, r_{c}=0.01$ and $r_{s}=0.1$, we find $P_{P N}=0.01438$ using the exact analytical expression and we find $P_{P N}=0.01562$ using (27). When $r_{c}=r_{s}=$ 0.01 , i.e. strong coupling and strong self-feedback, we have $P_{P N}=0.01356$ and approximation (28) gives 0.01367 .

Probabilities of oscillations as a function of the coupling ratio $r_{c}$ are plotted Fig. 7 for the three oscillators. Different values for the self feedback ratio are used. Probability of oscillations for the different models decreases with $r_{c}$ suggesting that strong negative coupling facilitate oscillations. The addition of a negative self-feedback reduces the chance to oscillate whereas a moderate positive selffeedback does not modify significantly the probability of oscillations as shown Fig. 8. The effect of a negative or a positive self-feedback loop is illustrated in 

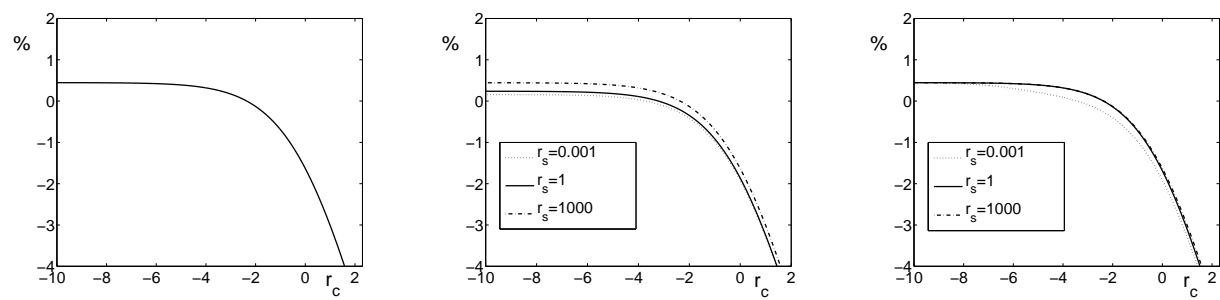

Figure 7: Log-log plot of the percentage of parameter sets that yield stable oscillations as a function of the coupling ratio, $r_{c}=k / k_{c}$, for the negative feedback-only oscillator (left panel), negative-plus-negative oscillator (middle panel) and positive-plus-negative oscillator (right panel). For the two last oscillators, the percentage is computed for different values of the self-feedback ratio $r_{s}=k / \bar{k}_{7}$.

details Fig. 9 where the probability is plotted as a function of $r_{s}$ for different $r_{c}$ values. It is shown that the probability of oscillations is an increasing function of $r_{s}$ and crucially depends on the coupling ratio $r_{c}$. As the self-feedback ratio $r_{s}$ increases a sigmoid-type transition occurs from a low-probability level to a higher probability level. The transitions for the PN and NN oscillators are compared Fig. 10. The high-probability level is reached when $r_{s} \rightarrow+\infty$ (weak self-feedback). This level can be maintained for a large range of $r_{s}$ values when the self-feedback loop is positive. However when $r_{c} \gg 1$ the difference induced by the nature of the self-feedback connection vanishes and the probability of oscillations becomes negligible.

The strong coupling regime $\left(r_{c} \ll 1\right)$ is related to the fast-slow repressilator previously discussed and is revealed to be the configuration that maximizes the chance to function in an oscillatory regime. Moreover when the strength of the self-feedback is also strong, we found, for $r_{s}=r_{c} \ll 1$,

$$
\begin{aligned}
& P_{N N}=\frac{1}{K^{3}}\left(1-\frac{1}{K}\right), \\
& P_{P N}=\frac{1}{K^{3}}\left(1-\frac{1}{4 K}\right)
\end{aligned}
$$

which has $P_{N N}=3^{3} / 4^{4} \approx 0.1055$ and $P_{P N}=3 / 4$ as a maximum when $K=4 / 3$ and $K=1$, respectively.

Figure 8 indicates that the high cooperativity regime gives an upper approximation of the probability of oscillations comparing to the smooth case for the the No-oscillator and NN-oscillator whereas a lower approximation is obtained for the PN-oscillator. 


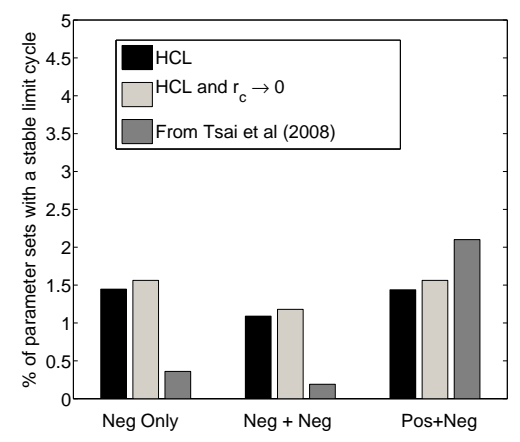

Figure 8: Percentage of parameter sets that yield stable oscillations. Each group is for an oscillator: the negative feedback-only oscillator (left group), the negative-plus-negative oscillator (middle group) and the positive-plus-negative oscillator (right group). The black bar is for the high cooperativity limit (HCL) with a coupling ratio $r_{c}=0.01$ and a self-feedback ratio $r_{s}=0.1$. The middle bar represents the maximum probability obtained as the coupling ratio $r_{c}$ tends towards 0 (strong coupling). The right bar represents the result of the numerical investigations in the smooth case.

\subsubsection{Helmholtz decomposition of the piecewise linear oscillators}

In the high cooperativity limit, the functions (5) and (6) can be simplified as follows

$$
\begin{aligned}
S_{i}^{[1]}(x) & =\left(x-K_{i}\right) \Theta\left(x-K_{i}\right), \\
S_{i}^{[2]}(x) & =\frac{1}{2}\left(x-K_{i}\right)^{2} \Theta\left(x-K_{i}\right)
\end{aligned}
$$

and the scalar potential (8) can be rewritten as quadratic function combined with step functions. It is straightforward to show that the potential functions of the oscillators admit a global minimim. Oscillations are therefore generated by a balance between an attracting fixed point where the gradient part vanishes and a rotating part derived from the potential vector

$$
G(A, B, C)=\left(\begin{array}{c}
k_{6} C\left(B-K_{3}\right) \Theta\left(B-K_{3}\right) \\
k_{2} A\left(C-K_{1}\right) \Theta\left(C-K_{1}\right) \\
k_{4} B\left(A-K_{2}\right) \Theta\left(A-K_{2}\right)
\end{array}\right)
$$

For the No-oscillator and for parameters satisfying (13), the minimum is given

$$
\left(A_{m}, B_{m}, C_{m}\right)=\left(\frac{k_{1}+k_{4} K_{2}}{k_{1}+k_{4}}, \frac{k_{3}+K_{3} k_{6}}{k_{3}+k_{6}}, \frac{k_{5}+K_{1} k_{2}}{k_{5}+k_{2}}\right) .
$$

If $K_{i}>1$, the corresponding minimum is 1 . Therefore the necessary condition for the existence of a limit cycle $K_{i}<1$ can be reformulated as : the minimum 

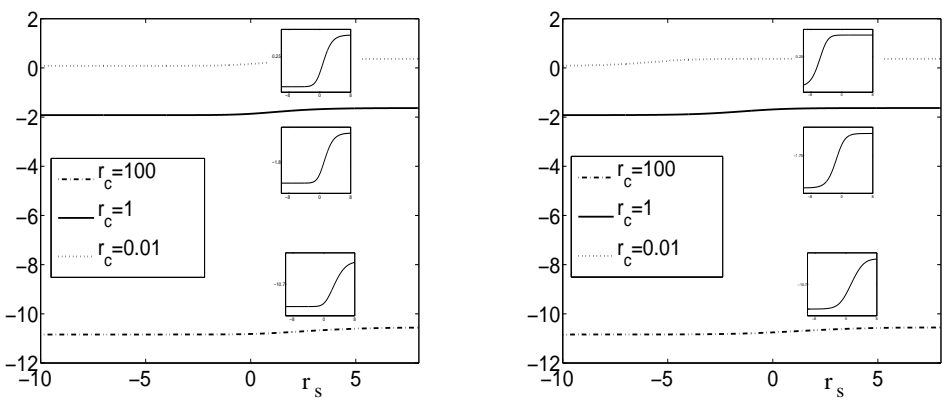

Figure 9: Log-log plot of the percentage of parameter sets that yield stable oscillations as a function of the self-feedback ratio, $r_{s}=k / \bar{k}_{7}$, for the negativeplus-negative oscillator (left panel) and positive-plus-negative oscillator (right panel). The percentage is computed for different values of the coupling ratio: $r_{c}=100, r_{c}=1$ and $r_{c}=0.01$. The insets are rescaling of the Y-axis of the corresponding curve (see also Fig. 10).

point of the potential energy has to be greater than the median effective concentration (component by component).

For the NN-oscillator, the additional term in the potential energy can be rewritten as

$$
\tilde{\phi}_{N N}(A)=\frac{k_{7}}{2}\left(A^{2}-K_{4}^{2}\right) \Theta\left(A-K_{4}\right) .
$$

The minimum point remains unchanged if $A_{m}<K_{4}$ and a sufficient condition is $K_{4}>1$ corresponding to the upper zone of existence of limit cycles (see Fig. $4 \mathrm{~B})$. In this case the minimum point $A_{m}$ is lower then the median effective concentration $K_{4}$ but gretaer than $K_{2}$. For the other set of parameter values yielded oscillations ( bottom left part of Fig. 4B), the minimum is given by

$$
A_{m}=\frac{k_{1}+k_{4} K_{2}}{k_{1}+k_{4}+k_{7}},
$$

and $B_{m}, C_{m}$ remain unchanged. Thus $A_{m}>K_{2}$ is equivalent to the necessary condition $K_{2}<K_{4, b}$ previously derived (when $K_{4}<1$ ).

For the PN-oscillator, we have

$$
\tilde{\phi}_{P N}(A)=\frac{k_{7}}{2}\left(A-K_{4}\right)\left(A+K_{4}-2\right) \Theta\left(A-K_{4}\right) .
$$

The minimum stays unchanged if $A_{m}<K_{4}$, otherwise the minimum becomes

$$
A_{m}=\frac{k_{1}+k_{7}+k_{4} K_{2}}{k_{1}+k_{4}+k_{7}} .
$$

In both cases $A_{m}>K_{2}$ reads $K_{2}<1$. 

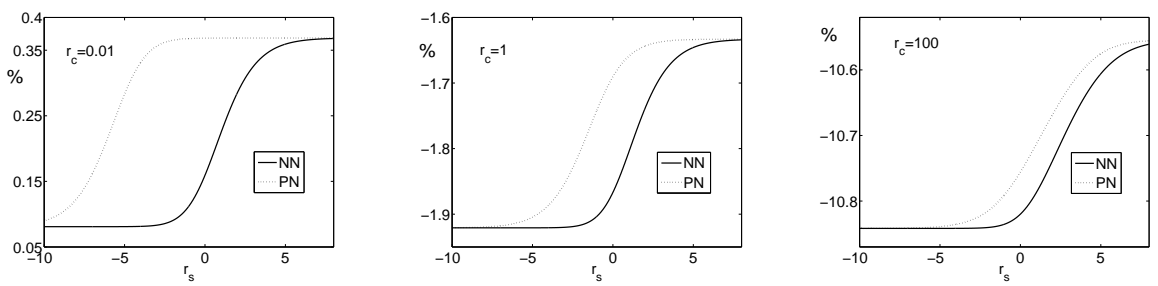

Figure 10: Log-log plot of the percentage of parameter sets that yield stable oscillations as a function of the self-feedback ratio for different values of the coupling ratio. The negative-plus-negative oscillator is compared with the positiveplus-negative oscillator for different values of the coupling ratio: $r_{c}=0.01$ (left), $r_{c}=1$ (middle) and $r_{c}=100$ (right).

\subsection{The smooth case: numerical analysis of oscillatory regimes}

In this section we will investigate numerically the smooth sigmoidal case and we will determine the domain of existence of stable oscillations in the parameter space together with the size and location of the limit cycles.

\subsubsection{Domain of oscillations}

We redid the numerical experiments of Tsai et al. (2008): for the different oscillators, we generated random parameter sets (see appendix C) until we had obtained 500 that gave stable oscillations. The distributions of parameters $\left(K_{i}\right)_{i=1, \ldots, 4}$ that give oscillations are represented with histograms shown in Fig. 11A, B and $\mathrm{C}$ for the three different oscillators. Figures $11 \mathrm{D}$ and $\mathrm{E}$ show the distributions in the $\left(K_{2}, K_{4}\right)$ plane for the NN- and PN-oscillator and Fig. $11 \mathrm{~F}, \mathrm{G}$ and $\mathrm{H}$ show the distributions of the Hill coefficients. Figure 11 indicates that there are no significative differences in the distribution of parameters between the No-oscillator ${ }^{3}$ and the NN-oscillator. The histograms of the median effective concentrations are peaked near a value slightly less than 1 (reminiscent to the condition for oscillations $K_{i}<1$ obtained for the step-like coupling) whereas the Hill coefficients are mainly located at large values. Therefore a high cooperativity facilitates oscillatory activities and explained the upper approximation of the probability of oscillations obtained when $n_{i} \gg 1$. The existence of a positive self-feedback loop makes more uniform the distributions of parameters indicating weak constraints on parameters for limit cycle existence. Parameter $K_{4}$ of the NN-oscillator has to be large (greater than 1) suggesting that the additional negative self-feedback loop is preferentially inactived during oscillations whereas the positive loop plays an enhancing role in oscillatory regimes

\footnotetext{
${ }^{3}$ The nonsymmetric distribution of parameters $\left(K_{i}\right)_{i=1,2,3}$ observed for the No-oscillator is due to the fact that $k_{5}$ has been fixed to 1 .
} 


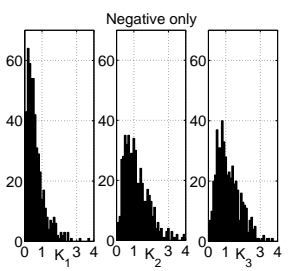

A

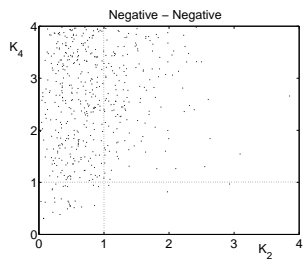

$D$

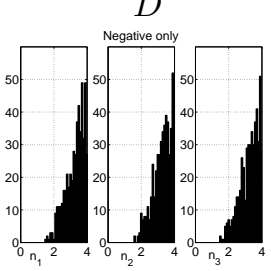

F

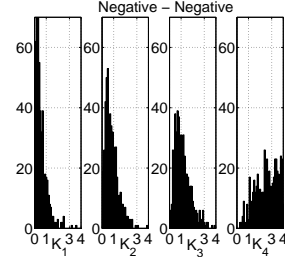

$B$

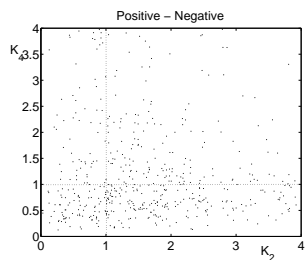

$E$

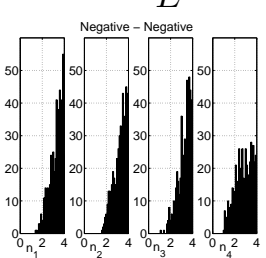

G

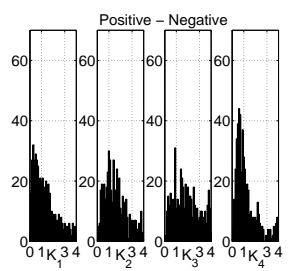

C

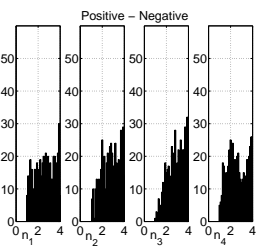

$H$

Figure 11: Distributions of parameters leading to oscillations for the different oscillator models. (A) Histograms of $K_{1}, K_{2} K_{3}$ for the negative feedback-only oscillator and of $K_{1}, K_{2}, K_{3}, K_{4}$ for (B) the negative-plus-negative oscillator and $(\mathrm{C})$ the positive-plus-negative oscillator. The histograms are computed for 500 parameter sets that gave oscillations for each oscillator. The projections of the distributions in the $\left(K_{2}, K_{4}\right)$ parameter space are shown panel (D) and (E) for the negative-plus-negative oscillator and for the positive-plus-negative oscillator, respectively. Histograms of the distributions of the Hill coefficients, $\left(n_{i}\right)_{i=1, \ldots, 4}$, are shown panels $(\mathrm{F}),(\mathrm{G})$ and $(\mathrm{H})$ (models as for $(\mathrm{A}),(\mathrm{B}),(\mathrm{C})$ respectively).

(histogram peaks for a value less than 1). For the PN-oscillator, a local peak of the $n_{4}$-histogram occurs close to $n_{4}=2$ suggesting that moderate $n_{4}$ values also facilitates oscillations and therefore explains the lower approximation previously computed in the high cooperativity limit.

\subsubsection{Location and size of limit cycles}

In order to assess the location and the size of the limit cycles we compute for the three different oscillators and for each variable of the model: (i) the mean value 
$\bar{X}=1 / T \int_{0}^{T} X(t) d t$ for $X=A(t), B(t)$ or $C(t)$ and (ii) the peak amplitude $|X|=\max X(t)-\min X(t)$ where $(A(t), B(t), C(t))$ is the limit cycle solution and $T$ the period of the oscillations. Results are shown Fig 12. It can be
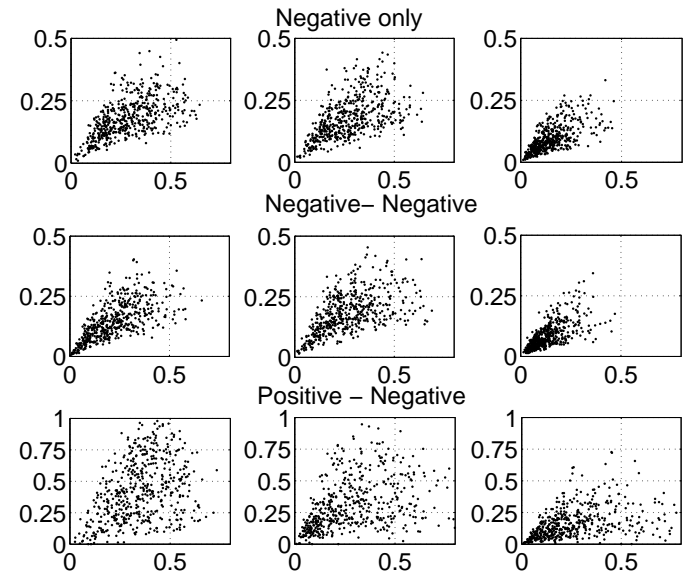

Figure 12: Plots of the peak amplitude as a function of the mean location of the limit cycles for the three different oscillators. The first row is for the oscillator without self feedback, the second one is for the negative-plus-negative oscillator and the third one is for the positive-plus-negative oscillator. The left column is for the $A$ variable, the middle one is for $B$ and the right one is for $C$. A different scale of the vertical axis has been used for the positive-plus-negative oscillator.

seen that the PN-oscillator supports a larger variability in the amplitude and location of its limit cycle whereas the No-oscillaor and NN-oscillator exhibits similar properties. This is also illustrated in Fig. 13 where some limit cycles are plotted in the phase space. A larger filling is obtained for the PN-oscillator. Results suggest that the addition of a positive self-feedback loop produces a higher variability and thus enhances the tunability of the system.

\subsection{A generalized repressilator-type model}

The core pathway motif that underpins sustained oscillations may involve more than three components. The generalization to an arbitrary number $N$ of chemical species is a cyclic biochemical feedback circuit where interactions are sequenced in series, schematically represented by $x_{1} \dashv x_{2} \dashv \ldots \dashv x_{N} \dashv x_{1}$. We will investigate the oscillatory behaviour of the following repressilator-type network

$$
\begin{aligned}
& \frac{d x_{1}}{d t}=k_{1}\left(1-x_{1}\right)-k_{2} x_{1} S_{1}\left(x_{N}\right) \\
& \frac{d x_{i}}{d t}=k_{2 i-1}\left(1-x_{i}\right)-k_{2 i} x_{i} S_{i}\left(x_{i-1}\right), \quad \text { for } \quad i=2, \ldots, N,
\end{aligned}
$$



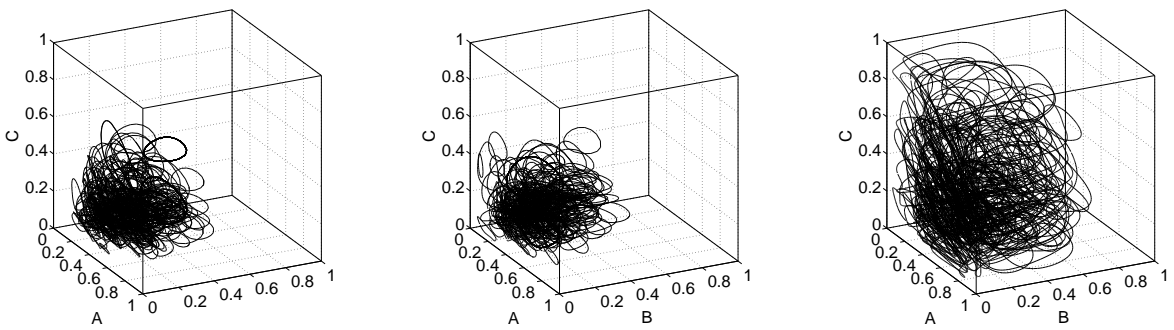

Figure 13: Plots of the limit cycles in the state space (250 limit cycles have been plotted) for the negative feedback-only oscillator (left), the negative-plusnegative oscillator (middle) and positive-plus-negative oscillator (right).

where $x_{i}$ is the concentration (or fraction of the concentration) of the chemical species $i, k_{2 i-1}$ and $k_{2 i}$ are kinetic constants, $K_{i}$ are the dissociation constants and $S_{i}$ are the sigmoidal functions given by (2). Here we restrict our attention to a network without self-feedback loop. Each species is repressed (inhibited) by the species immediately preceding in the loop. The network architecture is based on the successive inhibition of the chemical species and follows the design principles of the repressilator. A similar generalized model of repressilator-type has been proposed and studied in (Müller et al. (2006)).

For a switch-like coupling obtained in the limit of large Hill coefficients the equations become

$$
\frac{d x_{i}}{d t}=k_{2 i-1}\left(1-x_{i}\right)-k_{2 i} x_{i} \Theta\left(x_{i-1}-K_{i}\right), \quad \text { for } \quad i=1, \ldots, N,
$$

where we define $x_{0}=x_{N}$. Shifting the origin to the point of intersection of all thresholds, the system could be rewritten as

$$
\frac{d x_{i}}{d t}=a_{i}\left(x_{i}\right)-b_{i}\left(x_{i}\right) \operatorname{sgn}\left(x_{i-1}\right), \quad \text { for } \quad i=1, \ldots, N,
$$

where $a_{i}(x)=k_{2 i-1}-\left(k_{2 i-1}+k_{2 i} / 2\right)\left(x+K_{i+1}\right), b_{i}(x)=k_{2 i} / 2\left(x+K_{i+1}\right)$ and sgn is the sign function.

An analysis (not shown) similar to the one performed for the three-component oscillator gives

- if $N$ is odd, oscillatory regimes are obtained for

$$
\begin{aligned}
& K_{i}^{*}<K_{i}<1, \quad i=2, \ldots, N \text { where } \\
K_{i}^{*}= & \frac{k_{2 i-3}}{k_{2 i-3}+k_{2 i-2}},
\end{aligned}
$$

where $k_{0}=k_{2 N}$ and $k_{-1}=k_{2 N-1}$. 
- if $N$ is even, the network admits at least one stable fixed point. In particular, for parameters belonging to the domain of periodic behaviour obtained for the negative feedback system, i.e. (29), it can be shown that the network exhibits bistability between the two stable steady states

$$
\left(1, K_{3}^{*}, 1 \ldots K_{N-1}^{*}, 1, K_{1}^{*}\right) \quad \text { and } \quad\left(K_{2}^{*}, 1, K_{4}^{*} \ldots 1, K_{N}^{*}, 1\right)
$$

This situation is similar to the one encountered for the two-component system where it is easy to see that $\left(1, K_{1}^{*}\right)$ and $\left(K_{2}^{*}, 1\right)$ are two stable fixed points that coexist if and only if $K_{1}^{*}<K_{1}<1$ and $K_{2}^{*}<K_{2}<1$.

The qualitative difference in the dynamics between an odd and an even number of elements in the cycle has already been pointed out by Fraser and Tiwari (1974) and studied by Smith (1987) and subsequently in (Mallet-Paret and Smith (1990); Müller et al. (2006)) for similar cyclic feedback systems. It has been shown that this difference mainly manifests for strong nonlinearities whereas for weak nonlinearities the number of molecules involved in the loop does not play a crucial role in the dynamics. An even number of negative interactions canceled each other and the network essentially acts as a positive feedback system. The fundamental difference of both networks is related to the competitive nature of negative feedback systems whereas positive feedback systems are of cooperative and irreductible nature in the sense of Hirsch (Hirsch $(1982,1985))$. For cooperative systems, almost all trajectories tend to a steady state that always exists for $N$ even. When two stable fixed points coexist, the system is bistable and could function as a switch (Cherry and Adler (2000)).

For an odd number of nodes and for uniformly distributed parameters, i.e. $K_{i} \sim U\left(0, \bar{K}_{i}\right), i=1, \ldots, N$ and $k_{i} \sim U\left(0, \bar{k}_{i}\right), i=1, \ldots, 2 N$, the probability of oscillations is given by

$$
P_{N o}=\prod_{1}^{N} \frac{1}{2 \bar{K}_{i}}\left(1+F\left(\frac{\bar{k}_{2 i-2}}{\bar{k}_{2 i-3}}\right)-F\left(\frac{\bar{k}_{2 i-3}}{\bar{k}_{2 i-2}}\right)\right)
$$

where we define $\bar{k}_{0}=\bar{k}_{2 N}$ and $\bar{k}_{-1}=\bar{k}_{2 N-1}$. The function $F(x)$ is given by (19 ). For dissociation constants with identical maximal values, $\bar{K}_{i}=K$, and for identical ratios of maximum rate constants, $r_{c}=\bar{k}_{2 i-1} / \bar{k}_{2 i}$, we obtain

$$
P_{N o}=\frac{1}{2^{N} K^{N}}\left(1+F\left(r_{c}\right)-F\left(\frac{1}{r_{c}}\right)\right)^{N} .
$$

Probability (30) is represented in Fig. 14A as a function of $r_{c}$ for different $N$ values. In the limit of a strong coupling (or weak internal dynamics), i.e. $r_{c} \ll 1$, we obtain the following asymptotic expansion of the probability of oscillations

$$
P_{N o}=\frac{1}{K^{N}}+\frac{N r_{c}}{2 K^{N}} \ln \left(r_{c}\right)+r_{c} O(1) .
$$

Some typical trajectories of network elements are shown Fig.14B for a network of $N=19$ nodes with a weak $r_{c}$ value. The activity is characterized by sequential 

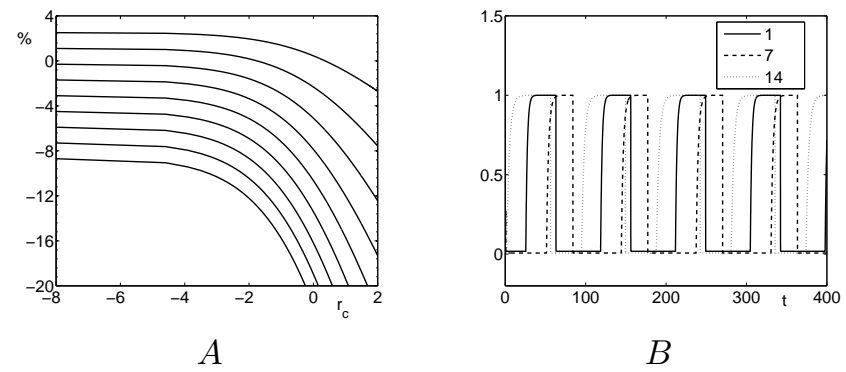

Figure 14: A. Log-log plot of the percentage of parameters leading to oscillation as a function of the coupling ratio $r_{c}$ for different lengths of the negative feedback oscillator. Probabilities obtained for odd $N$ values from $N=3$ (upper) to $N=19$ (lower) are depicted. We used $K=2$. B. Oscillations in a circular negative feedback system of $N=19$ nodes. Parameters $K_{i}, k_{2 i}$ and $k_{2 i}$ are uniformly distributed on $(0,1),(0,100)$ and $(0,1)$, respectively $\left(r_{c}=0.01\right)$. Activity of elements $i=1,7,14$ is represented (starting from a random intial condition in $\left.[0,1]^{N}\right)$.

successions of active and inactive states. Probability (30) vanishes as $N$ becomes large except for $K=1$ and for sufficiently small $r_{c}$ values. More precisely, a nonzero probability is reached if and only if $r_{c} N \ll 1$, i.e. $r_{c}$ has to be sufficiently small with respect to $N$, or equivalently a necessary and sufficient condition is $N r_{c}^{\beta}$ bounded $\forall \beta<1$.

\section{Discussion}

In the present work, we corroborated and characterized some observations already made or proved in various biological systems. (i) Oscillations are promoted by negative feedbacks. (ii) Strong nonlinearities facilitate oscillations: the more switch-like are the interactions, the easier it is to generate oscillations. (iii) A qualitative difference in the dynamics of monotone cyclic feedback systems occurs between an even or an odd number of components in the cycle. (iv) A strong inhibitory coupling induces a fast-slow dynamics that maximizes the probability of oscillations. In this regime, the dynamics is characterized by sequential switches from active to inactive states and vice-versa. (v) Small molecular circuits are better candidates to design biological oscillators. As the length $N$ of the feedback loop increases, the coupling strength $1 / r_{c}$ has to increase in order to maintain $N r_{c} \ll 1$. Therefore the claim 'the longer the loop, the easier it is to produce oscillations' (see for instance (Ferrell et al. (2011)) is not fully corroborated by our study. However we used here a restrictive framework where interactions are step-like that seems to be unlikely in many biological systems. It is suspected that a longer cycle allows to a relaxation of the assumption on the stiffness of nonlinearities (Smith (1987)) and a balance 
between 'moderate' Hill coefficients, sufficiently long feedback loop and strong inhibitory coupling should be reached to maximize the chance to oscillate.

Adding a negative self-feedback loop in a negative cyclic feedback oscillator does not present any new advantages whereas a moderate positive self-feedback weakens the constraints on parameters in the oscillatory regime and improves the flexibility of the system. Tunability induced by positive loops has already been observed in molecular systems (López-Caamal et al. (2013); Tsai et al. (2008)) and is essential in cases where cellular processes require a tight regulation. The additional self-feedback loop modifies the Helmholtz decomposition of the oscillators. The divergence-free part of the decomposition, analog to a circulating density responsible for a vortex-like behaviour, remains unchanged but the gradient part that minimizes the corresponding potential energy is affected. A positive self-feedback loop increases the divergence of the system and facilitates oscillations whereas a negative self-feedback loop tends to stabilize the system on a steady state precluding oscillations. However the Helmholtz decomposition does not yield any additional results but provides a new point of view on the structure of the flow of the 3-components negative feedback system. There are various ways of taking into account the fluctuations inherent in real systems. Two different frameworks have been used to address the issue of randomness and limit cycles: random environments (Lin and Kahn (1977)) and uncertain environnements (Falkenburg (1979)). Here we considered random parameters. The system remains deterministic and it is expected that stable solutions persist under random perturbations. However the presence of a stochastic term in the nonlinear differential system may induce new behaviours. A stochastic switching between two stable states has been observed in three-node genetic regulatory networks ( $\mathrm{Li}$ et al. (2012)). It has been shown that the unstable periodic solution of repressilator-type networks with an even number of nodes can be stabilized with random walk noise (Strelkowa and Barahona (2010)). This suggests that oscillations is more widespread than expected by the study of the deterministic system and the probability of oscillations are enhanced by a brownian noise.

Determining the conditions for robust oscillations has attracted a renewal of interest with the emergence of synthetic biology (Elowitz and Leibler (2000); Stricker et al. (2008); Gardner et al. (2000)). It has become possible to construct in the laboratory a biological system according to 'design specifications' derived from analytical and/or computational approaches. Networks of interacting species (mainly gene networks) are shaped in order to perform a given function, oscillation being the primary target due to its central role in cellular functions (Elowitz and Leibler (2000)). The calculation of the probability of having oscillations when fluctuating parameters are considered is of particular interest in this engineering-based approach. Despite the fact that parameters of a real biological system are probably not uniformely distributed with independent distribution, our approach suggests a way to tackle this issue. Moreover one of the macroscopic data directly available in the in-vivo implementation of synthetic oscillators is the percentage of oscillating cells that can, in turn, gives an estimation of the parameter distributions. 
The sufficient conditions for oscillations derived in the present study are based on steady states analysis. The strong correlation between the global dynamics and the properties of the system that arise by steady state analysis has already been pointed out by Smith (1987). The striking fact is that the local study inherent in fixed point analysis reveals the global dynamics of the system. The sufficient conditions thus obtained are necessary if we assume that the existence of a stable steady state precludes the existence of a stable limit cycle. It is known that such a bistability-exclusion can be broken by additional interactions that modify the cyclic nature or the monotonicity of the model and allow for multistability between a fixed point and a limit cycle, chaotic solutions or dynamics not allowed in $R^{2}$. For instance bistability may occur in two-side interaction systems, i.e. there exists $j$ such that $\dot{x}_{j}=f_{j}\left(x_{j}, x_{j-1}, x_{j+1}\right)$ (Li et al. (2012)). However due to the generalization of the Poincaré-Bendixson theorem of Mallet-Paret and Smith (1990) to the two-sided interaction system, chaotic solutions are not allowed (Elkhader (1992)). If the monotonicity property fails then chaotic solutions may appear (Cera et al. (1989)). Moreover a subcritical Hopf bifurcation generating bistability can be obtained in monotone negative feedback systems with a variable self-feedback loop (Hasty et al. (2002)) indicating that bistability-exclusion probably requires monotonicity conditions on the self-interaction term. However bistability often occurs in a narrow region of parameter space (see Hasty et al. (2002) for instance) and one can expect that the probability of oscillations derived from steady state analysis would be a good approximation.

We did not consider explicitly delay, an important element present in many biological systems. It is suspected that an effective form of time delay is, in many situations, only the result of a cascade of reactions and cellular processes that occur in the cell and therefore are implicitly taken into account by the cyclic structure of the model (Stricker et al. (2008)). Many properties obtained for monotone cyclic feedback systems also hold in the presence of delays and similar dynamics is expected (Mahaffy (1980); Smith (1987); Mallet-Paret and Sell (1996)).

\section{A The Jacobian matrix}

For the negative feedback-only oscillator, the jacobian matrix is given by

$$
J_{N o}=\left(\begin{array}{ccc}
-k_{1}-k_{2} S_{1}(C) & 0 & -\frac{k_{2} n_{1} K_{1}^{n_{1}} C^{n_{1}-1}}{\left(K_{1}^{n_{1}}+C^{n_{1}}\right)^{2}} A \\
-\frac{k_{4} n_{2} K_{2}^{n_{2}} A^{n_{2}-1}}{\left(K_{2}^{n_{2}}+A^{n_{2}}\right)^{2}} B & -k_{3}-k_{4} S_{2}(A) & 0 \\
0 & -\frac{k_{6} n_{3} K_{3}^{n_{3}} B^{n_{3}-1}}{\left(K_{3}^{n_{3}}+B^{n_{3}}\right)^{2}} C & -k_{5}-k_{6} S_{3}(B)
\end{array}\right) .
$$

where it is easy to see that each term is negative. For the NN and PN oscillators only the term in the first row and first column differs and we have

$$
\left(J_{N N}\right)_{11}=-k_{1}-k_{2} S_{1}(C)-k_{7} \frac{\left(n_{4}+1\right) K_{4}^{n_{4}} A^{n_{4}}+A^{2 n_{4}}}{\left(K_{4}^{n_{4}}+A^{n_{4}}\right)^{2}}
$$


that is always negative and

$$
\left(J_{P N}\right)_{11}=-k_{1}-k_{2} S_{1}(C)+k_{7} \frac{n_{4} K_{4}^{n_{4}} A^{n_{4}-1}-\left(n_{4}+1\right) K_{4}^{n_{4}} A^{n_{4}}-A^{2 n_{4}}}{\left(K_{4}^{n_{4}}+A^{n_{4}}\right)^{2}}
$$

which has a positive term. Other components are given by $\left(J_{N N}\right)_{i j}=\left(J_{P N}\right)_{i j}=$ $\left(J_{N o}\right)_{i j}$ for $(i, j) \neq(1,1)$

\section{B The piecewise linear oscillators}

Our analysis is based on the observation that each parameter $K_{i}$ defines a threshold plane that divises the phase space into rectangular boxes, the socalled regulatory domains. Inside each regulatory domain the system is linear and the analysis is straightforward.

\section{B.1 Fixed points}

The three different oscillators may admit fixed points depending on parameter values. Due to the discontinuity of the vector field, it is convenient to distinguish between two classes of fixed points (Glass and Pasternack (1978a); Mestl et al. (1995a); Snoussi and Thomas (1993)): 'regular' steady points and 'singular' steady points. Regular steady points are defined following the well-established theory of smooth dynamical systems. Singular steady states are characterized by the fact that at least one of its components lies on a threshold and thus require a specific treatment (Filippov (1988)).

Let $X_{s s}=\left(A_{s s}, B_{s s}, C_{s s}\right)$ be a fixed point. The regular fixed points of the No-oscillator and the corresponding conditions of existence are given by

$$
\begin{aligned}
& A_{s s}=1 \quad \text { for } \quad C_{s s}<K_{1} \text { and } A_{s s}=K_{2}^{*} \text { otherwise, } \\
& B_{s s}=1 \quad \text { for } A_{s s}<K_{2} \text { and } B_{s s}=K_{3}^{*} \text { otherwise, . } \\
& C_{s s}=1 \text { for } B_{s s}<K_{3} \text { and } C_{s s}=K_{1}^{*} \text { otherwise. }
\end{aligned}
$$

It is easy to show that when a regular fixed point exists it is stable. Each species, $A, B$ and $C$, can formally admit two different values at its resting state and thus we can distinguish between eight analytically different regular fixed points. The different possible steady states are the so-called focal points (Glass and Pasternack (1978a,b); Mestl et al. (1995a)) of the associated regulatory domain. If the focal point is inside its regulatory domain, it is a stable steady state of the system. Otherwise the system will leave the current regulatory domain and enter a new one that may have a different focal point.

The steady state $X_{s s}$ is a singular fixed point if $0 \in \mathcal{F}\left(X_{s s}\right)$ where $\mathcal{F}$ is the multi-valued function obtained when the Heaviside function of the component value that lies on its threshold is allowed to vary in $(0,1)$. For the No-oscillator, the only singular fixed point is $\left(K_{2}, K_{3}, K_{1}\right)$ that exists when a solution $\left(\Theta_{A}, \Theta_{B}, \Theta_{C}\right) \in[0,1]^{3}$ can be found to the following system

$k_{1}\left(1-K_{3}\right)-k_{2} K_{3} \Theta_{C}=0, k_{3}\left(1-K_{3}\right)-k_{2} K_{3} \Theta_{A}=0, k_{5}\left(1-K_{3}\right)-k_{2} K_{3} \Theta_{B}=0$. 
We obtain the conditions

$$
K_{1}^{*}<K_{1}<1, K_{2}^{*}<K_{2}<1, K_{3}^{*}<K_{3}<1,
$$

that (as we will show hereinafter) coincide with the conditions for oscillations. The state $\left(K_{2}, K_{3}, K_{1}\right)$ is the so-called loop characteristic state (Snoussi and Thomas (1993)) of the No-oscillator. We show in appendix B.4 that this singular fixed point is always unstable.

For the NN-oscillator, the values and the conditions for the existence of regular fixed points are identical to those obtained in the No-oscillator model for the two components $B_{s s}$ and $C_{s s}$. For $A_{s s}$ we get

$$
\begin{array}{ccccc}
A_{s s}=1 & \text { for } & C_{s s}<K_{1} & \text { and } & K_{4}>1, \\
A_{s s}=K_{4, b}^{*} & \text { for } & C_{s s}<K_{1} & \text { and } & K_{4}<K_{4, b}^{*}, \\
A_{s s}=K_{2}^{*} & \text { for } & C_{s s}>K_{1} & \text { and } & K_{4}>K_{2}^{*}, \\
A_{s s}=K_{4, a}^{*} & \text { for } & C_{s s}>K_{1} & \text { and } & K_{4}<K_{4, a}^{*},
\end{array}
$$

and, as for the No-oscillator, a regular fixed point, when it exists, is stable. Singular steady points with at least one component on a discontinuity plane may exist. As previously the loop characteristic state $\left(K_{2}, K_{3}, K_{1}\right)$ is a singular steady state, and for $K_{4}>K_{2}$, the conditions of existence remain the same than for the No-oscillator. If $K_{4}<K_{2}$ conditions of existence become

$$
K_{1}^{*}<K_{1}<1, K_{4, a}^{*}<K_{2}<K_{4, b}^{*}, K_{3}^{*}<K_{3}<1 .
$$

We show in appendix B.4 that this singular fixed point is always unstable. Moreover an additional singular fixed point may occur on the discontinuity plane $A_{s s}=K_{4}$. This singular fixed point is induced by the additional selffeedback loop and defines a second loop characteristic state of the oscillator. It is easy to show that this steady-state exists and is stable for $K_{4, b}^{*}<K_{4}<1$ and $C_{s s}<K_{1}$ or for $K_{4, a}^{*}<K_{4}<1$ and $C_{s s}>K_{1}$. The A-component of the steady-state, $A_{s s}$, can formally take five different values, so that we distinguish between twenty analytically different stable fixed points for the NN-oscillator.

For the PN-oscillator, the steady state values $B_{s s}$ and $C_{s s}$ and the corresponding conditions of existence remain the same. For $A_{s s}$ we obtain the following values and conditions of existence

$$
\begin{aligned}
& A_{s s}=1 \text { for } \quad C_{s s}<K_{1} \text {, } \\
& A_{s s}=K_{2}^{*} \quad \text { for } \quad C_{s s}>K_{1} \quad \text { and } \quad K_{4}>K_{2}^{*} \text {, } \\
& A_{s s}=K_{4}^{*} \quad \text { for } \quad C_{s s}>K_{1} \quad \text { and } \quad K_{4}<K_{4}^{*} \text {. }
\end{aligned}
$$

The singular fixed point $\left(K_{2}, K_{3}, K_{1}\right)$ when it exists is unstable (see appendix B.4). Moreover, as for the NN-oscillator, the additional self-feedback loop may induce the existence of a singular fixed point on the discontinuity plane $A_{s s}=$ $K_{4}$. It is easy to show that this singular fixed point is always unstable. To sum up, the PN-oscillator has twelve different forms of stable steady states. It is worth noting that for $C_{s s}>K_{1}$ the two stable fixed points $A_{s s}=K_{2}^{*}$ and $A_{s s}=K_{4}^{*}$ can coexist when $K_{2}^{*}<K_{4}<K_{4}^{*}$. 
To summarize, when a regular fixed point exists, it is stable. In addition, singular fixed points may exist but are unstable except the singular fixed point of the NN-oscillator satisfying $A_{s s}=K_{4}$. It is worth mentioning that, for a given set of parameters, a stable fixed point, when it exists, is unique except for the PN-oscillator where two stable fixed points may coexist.

\section{B.2 Oscillations}

Based on the fixed points analysis done in Appendix B.1 it is possible to derive analytically the conditions on parameters of the system for the existence of stable fixed points. These conditions are monitored by the relative location between $K_{i}$, the unity, and the associated critical value $K_{i}^{*}$. For instance, for the No-oscillator, it is easy to check that when $K_{1}<K_{1}^{*}, K_{2}>K_{2}^{*}$ and $K_{3}<1$, the point $\left(K_{2}^{*}, 1, K_{1}^{*}\right)$ is a stable fixed point. It is thus possible to derive exactly the sets of parameters for which there are no stable fixed points taking the complementary of the sets for which stable fixed points exist. These sets are given by (13), (14) and (15) for the three different oscillators, respectively. Since a trajectory cannot escape from the box $D=[0,1]^{3}$, an oscillatory pattern exists inside the box. It is suspected that this oscillatory activity corresponds to a limit cycle (see the discussion in the section "Remarks on the oscillatory activities").

\section{B.3 Calculation of $P\left(K_{2}<K_{4, a}^{*}\right)$}

We can easely check that

$$
P\left(K_{2}<K_{4, a}^{*}\right)=\frac{1}{\bar{K}_{2} \bar{k}_{1} \bar{k}_{2} \bar{k}_{7}}\left(G\left(\bar{k}_{2}+\bar{k}_{7}\right)-G\left(\bar{k}_{7}\right)-G\left(\bar{k}_{2}\right)+G(0)\right)
$$

where

$$
G(u)=\int_{0}^{\bar{k}_{1}} x(x+u) \ln (x+u) d x .
$$

Integrating by parts we obtain

$$
G(u)=\frac{\left(\bar{k}_{1}+u\right)^{2}}{3}\left(\bar{k}_{1}-\frac{u}{2}\right) \ln \left(\bar{k}_{1}+u\right)+\frac{u^{3}}{6} \ln (u)-\frac{\bar{k}_{1}}{36}\left(3 \bar{k}_{1} u-6 u^{2}+4 \bar{k}_{1}^{2}\right)
$$

that completes the analytical expression of $P\left(K_{2}<K_{4, a}^{*}\right)$.

Let $r_{c}=k_{1} / k_{2}$ and $r_{s}=k_{1} / k_{7}$, analytical calculations give

$$
\begin{aligned}
P\left(K_{2}<K_{4, a}^{*}\right)= & \frac{1}{K}\left(\frac{1}{3}+\frac{r_{s}}{6 r_{c}^{2}} \ln r_{c}+\frac{r_{c}}{6 r_{s}^{2}} \ln r_{s}+\frac{r_{c} r_{s}}{6}\left(\frac{1}{r_{c}}+\frac{1}{r_{s}}\right)^{3} \ln \left(\frac{1}{r_{c}}+\frac{1}{r_{s}}\right)\right. \\
& +\frac{r_{s}}{3}\left(\frac{1}{r_{c}}+1\right)^{2}\left(\frac{1}{2}-r_{c}\right) \ln \left(1+\frac{1}{r_{c}}\right)+\frac{r_{c}}{3}\left(\frac{1}{r_{s}}+1\right)^{2}\left(\frac{1}{2}-r_{s}\right) \ln \left(1+\frac{1}{r_{s}}\right) \\
& \left.-\frac{1}{3}\left(\frac{1}{r_{c}}+\frac{1}{r_{s}}+1\right)^{2}\left(\frac{r_{s}}{2}+\frac{r_{c}}{2}-r_{c} r_{s}\right) \ln \left(1+\frac{1}{r_{c}}+\frac{1}{r_{s}}\right)\right)
\end{aligned}
$$




\section{B.4 Stability of the origin}

For the No-oscillator, the stability of singular steady state $\left(K_{2}, K_{3}, K_{1}\right)$ is determined by the stability of the origin of

$$
\begin{aligned}
\dot{x} & =k_{1}\left(1-K_{2}-x\right)-k_{2}\left(x+K_{2}\right) \Theta(z), \\
\dot{y} & =k_{3}\left(1-K_{3}-y\right)-k_{4}\left(y+K_{3}\right) \Theta(x), \\
\dot{z} & =k_{5}\left(1-K_{1}-z\right)-k_{6}\left(z+K_{1}\right) \Theta(y) .
\end{aligned}
$$

which is given by the study of the trajectories of the system

$$
\begin{aligned}
\dot{x} & =k_{1}\left(1-K_{2}\right)-k_{2} K_{2} \Theta(z), \\
\dot{y} & =k_{3}\left(1-K_{3}\right)-k_{4} K_{3} \Theta(x), \\
\dot{z} & =k_{5}\left(1-K_{1}\right)-k_{6} K_{1} \Theta(y) .
\end{aligned}
$$

We define

$$
\begin{aligned}
\alpha_{i} & =k_{2 i-1}\left(1-K_{i+1}\right), \\
\beta_{i} & =\left(k_{2 i-1}+k_{2 i}\right) K_{i+1}-k_{2 i-1},
\end{aligned}
$$

for $i=1,2,3$ where we set here $K_{4}=K_{1}$ for conveniance. Conditions for the existence of a singular fixed point at the origin lead to $\alpha_{i}>0$ and $\beta_{i}>0$. The dynamics can be rewritten as

$$
\begin{aligned}
& \dot{x}=\alpha_{1} \text { if } z<0 \text { and }-\beta_{1} \text { otherwise } \\
& \dot{y}=\alpha_{2} \text { if } x<0 \text { and }-\beta_{2} \text { otherwise } \\
& \dot{z}=\alpha_{3} \text { if } x<0 \text { and }-\beta_{3} \text { otherwise. }
\end{aligned}
$$

and we have $\operatorname{sgn}(\dot{x})=-\operatorname{sgn}(z), \operatorname{sgn}(\dot{y})=-\operatorname{sgn}(x)$ and $\operatorname{sgn}(\dot{z})=-\operatorname{sgn}(y)$. Note that a similar system is studied in (Farcot and Gouzé (2009)) but with different assumptions on the parameters. Here we will show using basic calculus that the 'local' system is unstable.

The trajectories make revolutions around the origin and pass many times into the plane $x=0$, intersecting it for $y>0$ (and $z<0$ ) and for $y<0$ (and $z>0$ ) for one revolution. After possibly a transient, the sign of the components defining the trajectory will follow the cycle $(+,+,-) \rightarrow(+,-,-) \rightarrow(+,-,+) \rightarrow$ $(-,-,+) \rightarrow(-,+,+) \rightarrow(-,+,-)$ (see figure $(6)$ where 1 corresponds to + and 0 to -$)$.

The trajectory of the system defines a mapping of the half plane $P_{0}\left(x_{0}=0\right.$, $\left.y_{0}>0, z_{0}<0\right)$ into itself. The solution with initial value $x_{0}=0, y_{0}>0, z_{0}<0$ lies first in the region (110) i.e. $(x>0, y>0, z<0)$, where the solution has the form

$$
x(t)=\alpha_{1} t, \quad y(t)=y_{0}-\beta_{2} t, \quad z(t)=z_{0}-\beta_{3} t .
$$

The trajectory intersects the plane $y=0$ at time $t_{a}=y_{0} / \beta_{2}$ at the point

$$
x_{a}=\alpha_{1} / \beta_{2} y_{0}, \quad y_{a}=0, \quad z_{a}=z_{0}-\beta_{3} / \beta_{2} y_{0},
$$


and enters into the domain 100. Using similar arguments, we compute the different reaching times, $t_{a}, \ldots, t_{f}$, of the different regions $(101,001,011,010$ and 110, respectively) together with the corresponding intersection points. We find that from the point $x_{0}=0, y_{0}>0, z_{0}<0$ in $P_{0}$ the trajectory first goes back into $P_{0}$ at the point $x_{f}=0, y_{f}, z_{f}$ where

$$
\left(\begin{array}{l}
y_{f} \\
z_{f}
\end{array}\right)=\left(\begin{array}{ll}
a_{11} & a_{12} \\
a_{21} & a_{22}
\end{array}\right)\left(\begin{array}{l}
y_{0} \\
z_{0}
\end{array}\right)
$$

with

$$
\begin{aligned}
& a_{11}=3+u_{1}+u_{2}+u_{3}+u_{1} u_{2}+u_{1} u_{3}+u_{2} u_{3}+u_{1} u_{2} u_{3}+\frac{1}{u_{1}}+\frac{1}{u_{3}}+\frac{1}{u_{1} u_{3}}, \\
& a_{12}=-\frac{\beta_{2}}{\beta_{3}}\left(2+u_{1}+u_{2}+u_{1} u_{2}+\frac{1}{u_{1}}+\frac{1}{u_{3}}+\frac{1}{u_{1} u_{3}}\right) \\
& a_{21}=-\frac{\beta_{3}}{\beta_{2}}\left(1+u_{3}+\frac{u_{3}}{u_{2}}+\frac{2}{u_{2}}+\frac{1}{u_{1} u_{2}}+\frac{1}{u_{2} u_{3}}+\frac{1}{u_{1} u_{2} u_{3}}\right), \\
& a_{22}=1+\frac{1}{u_{2}}+\frac{1}{u_{1} u_{2}}+\frac{1}{u_{2} u_{3}}+\frac{1}{u_{1} u_{2} u_{3}},
\end{aligned}
$$

where we set $u_{i}=\alpha_{i} / \beta_{i}$.

Equation (33) defines a 2D-linear mapping. Let $\lambda_{1}$ and $\lambda_{2}$ be the two associated eigenvalues. Since we have $\left|\lambda_{1}\right|+\left|\lambda_{2}\right| \geq\left|\lambda_{1}+\lambda_{2}\right|=\left|a_{11}+a_{22}\right|>4$ then at least one eigenvalue is greater than 1 in absolute value. Therefore, the origin is unstable. Numerical investigations suggest than one eigenvalue is large whereas the other is less than one, in absolute value, indicating a saddle configuration and the existence of a stable manifold associated with the origin.

For the two others oscillators, the NN-oscillator and the PN-oscillator, the situation remains the same for $K_{4}>K_{2}$. When $K_{4}<K_{2}$ we define $\alpha_{1, N N}=\alpha_{1}-$ $k_{7} K_{2}$ and $\beta_{1, N N}=\beta_{1}+k_{7} K_{2}$ for the NN-oscillator and $\alpha_{1, P N}=\alpha_{1}+k_{7}\left(1-K_{2}\right)$ and $\beta_{1, P N}=\beta_{1}-k_{7}\left(1-K_{2}\right)$ for the PN-oscillator. Conditions for the existence of the singular fixed point $\left(K_{2}, K_{3}, K_{1}\right)$ give $\alpha_{1, N N}>0, \beta_{1, N N}>0$ and $\alpha_{1, P N}>0, \beta_{1, P N}>0$. Study of stability proceeds along the same lines than for the No-oscillator and we show that the origin is unstable.

\section{Choice of the random parameter sets}

For the numerical simulation of the smooth oscillators we used the same parameter distributions as in (Tsai et al. (2008)). All parameters are dimensionless and $(\mathrm{H})$ holds (see (21)) with:

- $K=4$, i.e. we used $K_{i} \sim \mathcal{U}(0,4), i=1,2,3,4$,

- $k=10$, i.e. we used $k_{1}, k_{3} \sim \mathcal{U}(0,10)$,

- $k_{c}=1000$; i.e. we used $k_{2}, k_{4}, k_{6} \sim \mathcal{U}(0,10)$ 
except for $k_{5}$ that has been fixed to 1 . For the NN-oscillator and PN-oscillator, we used $k_{7} \sim U(0,100)$. Each Hill coefficient follows an uniform distribution over $(1,4)$.

\section{References}

Acary, V., Jong, H., Brogliato, B., 2013. Numerical simulation of piecewiselinear models of gene regulatory networks using complementarity systems. Physica D in press.

Angeli, D., Ferrell, J.E., Sontag, E.D., 2004. Detection of multistability, bifurcations, and hysteresis in a large class of biological positive-feedback systems. Proc. Natl. Acad. Sci. USA 101, 1822-1827.

Boulier, F., Lefranc, M., Lemaire, F., Morant, P.E., Ürgüplü, A., 2007. On proving the absence of oscillations in models of genetic circuits, in: Anai, H., Horimoto, K., Kutsia, T. (Eds.), Proceedings of Algebraic Biology 2007, Springer Verlag Berlin Heidelberg. pp. 66-80.

Buse, O., Kuznetsov, A., Pérez, R., 2009. Existence of limit cycles in the repressilator equations. Int. J. Bifurcat. Chaos 19, 4097-4106.

Buse, O., Pérez, R., Kuznetsov, A., 2010. Dynamical properties of the repressilator model. Phys. Rev. E 81. doi:10.1103/PhysRevE.81.066206.

Cera, E.D., Phillipson, P.E., Wyman, J., 1989. Limit-cycle oscillations and chaos in reaction networks subject to conservation of mass. Proc. Natl. Acad. Sci. USA 86, 142-146.

Cherry, J.L., Adler, F.R., 2000. How to make a biological switch. J. Theor. Biol. 203, 117-113.

Ciliberto, A., Novak, B., Tyson, J.J., 2005. Steady states and oscillations in the p53/ mdm2 network. Cell Cycle 4, 488-493.

Cinquin, O., Demongeot, J., 2002. Positive and negative feedback: striking a balance between necessary antagonists. J. Theor. Biol. 216, 229-241.

Demongeot, J., Glade, N., Forest, L., 2007a. Liénard systems and potentialhamiltonian decomposition i - methodology. C. R. Acad. Sci. Paris, Ser. I $344,121-126$.

Demongeot, J., Glade, N., Forest, L., 2007b. Liénard systems and potentialhamiltonian decomposition ii - algorithm. C. R. Acad. Sci. Paris, Ser. I 344, $191-194$.

Dokoumetzidis, A., Iliadis, A., Macheras, P., 2001. Nonlinear dynamics and chaos theory: concepts and applications relevant to pharmacodynamics. Pharm. Res. 18, 415-426. 
Domijan, M., Pécou, E., 2012. The interaction graph structure of mass-action reaction networks. J. Math. Biol. 65, 375-402.

Elkhader, A.S., 1992. A result on a feedback system of ordinary differential equations. J. Dyn. Differ. Equ. 4, 399-418.

Elowitz, M.B., Leibler, S., 2000. A synthetic oscillatory network of transcriptional regulators. Nature 403, 335-338.

Falkenburg, D.R., 1979. Existence of limit cycles in a non linear dynamic system with random parameters. WSC '79 Proceedings of the 11th conference on Winter simulation 1, 159-164.

Farcot, E., Gouzé, J.L., 2009. Periodic solutions of piecewise affine gene network models with non uniform decay rates: the case of a negative feedback loop. Acta Biotheoretica 57, 429-455.

Farcot, E., Gouzé, J.L., 2010. Limit cycles in piecewise-affine gene network models with multiple interaction loops. International Journal of Systems Science 41, 119-130.

Ferrell, J.E., Tsai, T.Y., Yang, Q., 2011. Modeling the cell cycle: why do certain circuits oscillate? Cell 144, 874-885.

Filippov, A.F., 1988. Differential equations with discontinuous righthand sides. Dordrecht, Kluwer Academic Publishers.

Fraser, A., Tiwari, J., 1974. Genetic feedback-repression. ii. cyclic genetic system. J. Theor. Biol. 47, 397-412.

Gardner, T.S., Cantor, C.R., Collins, J.J., 2000. Construction of a genetic toggle switch in escherichia coli. Nature 403, 339-342.

Gedeon, T., 1998. Cyclic feedback systems. Mem Am Math Soc 134.

Gedeon, T., Mischaikow, K., 1995. Structure of global attractor of cyclic feedback systems. J Dyn Differ Equ 7, 141-190.

Glass, L., Kaufman, S., 1973. The logical analysis of continuous non-linear biochemical control networks. J. Theor. Biol. 39, 103-129.

Glass, L., Pasternack, J., 1978a. Prediction of limit cycles in mathematical models of biological oscillations. B. Math. Biol. 40, 27-44.

Glass, L., Pasternack, J., 1978b. Stable oscillations in mathematical models of biological control systems. J. Math. Biol. 6, 207-223.

Goldbeter, A., 1991. A minimal cascade model for the mitotic oscillator involving cyclin and cdc2 kinase. Proc. Natl. Acad. Sci. USA 88, 9107-9111.

Goldbeter, A., 2002. Computational approaches to cellular rhythms. Nature 420, 238-245. 
Gouzé, J.L., 1998. Positive and negative circuits in dynamical systems. J. Biol. Sys. 6, 11-15.

Gouzé, J.L., Sari, T., 2003. A class of piecewise linear differential equations arising in biological models. Dynam Syst 17, 299-316.

Griffith, J., 1968. Mathemaics of cellular control processes. i. negative feedback to one gene. J. Theor. Biol. 20, 202-208.

Guantes, R., Poyatos, J., 2006. Dynamical principles of two-component genetic oscillators. PLoS Comput Biol 2. doi:10.1371/journal.pcbi.0020030.

Harris, S.L., Levine, A.J., 2005. The p53 pathway: positive and negative feedback loops. Oncogene 24, 2899-2908.

Hastings, S., Tyson, J., Webster, D., 1977. Existence of periodic solutions for negative feedback cellular control systems. J Differ Equations 25, 39-64.

Hastings, S.P., 1977. On the uniqueness and global asymptotic stability of periodic solutions for a third order system. Rocky Mt J Math 7, 513-538.

Hasty, J., Dolnik, M., Rottschäfer, V., Collins, J.., 2002. Synthetic gene network for entraining and amplifying cellular oscillations. Phys. Rev. Lett. 88. doi:148101.

Hirata, H., Yoshiura, S., Ohtsuka, T., Bessho, Y., Harada, T., Yoshikawa, K., Kageyama, R., 2002. Oscillatory expression of the bhlh factor hes1 regulated by a negative feedback loop. Science $298,840-843$.

Hirsch, H., 1982. Systems of differential equations which are competitive and cooperative. i. limit sets. SIAM J. Math. Anal. 13, 167-179.

Hirsch, H., 1985. Systems of differential equations which are competitive and cooperative. ii. convergence almost everywhere. SIAM J. Math. Anal. 16, $432-439$.

Hornung, G., Barkai, N., 2008. Noise propagation and signaling sensitivity in biological networks: a role for positive feedback. PLoS Comput Biol 4. doi:10.1371/journal.pcbi.0040008.

Ironi, L., Panzeri, L., Plahte, E., Simoncini, V., 779-794. Dynamics of actively regulated gene networks. Physica D 240.

de Jong, H., Geiselmann, J., Hernandez, C., Page, M., 2003. Genetic network analyzer: qualitative simulation of genetic regulatory networks. Bioinformatics $19,336-344$.

Kaufman, M., Soulé, C., Thomas, R., 2007. A new necessary condition on interaction graphs for multistationarity. J. Theor. Biol. 248, 675-685. 
Keener, J., Sneyd, J., 1998. Mathematical physiology. I: Cellular Physiology. Interdisciplinary Applied Mathematics 8, Springer.

Kuznetsov, Y., 2004. Elements of applied bifurcation theory. Applied Mathematical Sciences. New York: Springer.

Li, W., Krishna, S., Pigolotti, S., Mitarai, N., Jensen, M.H., 2012. Switching between oscillations and homeostasis in competing negative and positive feedback motifs. J. Theor. Biol. 307, 205-210.

Lin, J., Kahn, P.B., 1977. Limit cycles in random environments. SIAM J. Appl. Math. 32, 260-291.

López-Caamal, F., Middleton, R.H., Huber, H.J., 2013. Equilibria and stability for a class of positive feedback loops. J. Math. Biol. (in press).

Lu, L., Edwards, R., 2010. Structural principles for periodic orbits in glass networks. J. Math. Biol. 60, 513-541.

Machina, A., Edwards, R., van den Driessche, P., 2013. Singular dynamics in gene network models. SIAM J. on App. Dyn. Syst. 12, 95-125.

Mahaffy, J.M., 1980. Periodic solutions for certain protein synthesis models. J. Math. Anal. Appl. 74, 72-105.

Mallet-Paret, J., Sell, G.R., 1996. The poincaré-bendixson theorem for monotone cyclic feedback systems with delay. J. Differ. Equations 125, 441-489.

Mallet-Paret, J., Smith, H.L., 1990. The poincaré-bendixson theorem for monotone cyclic feedback systems. J. Dyn. Differ. Equ. 2, 367-421.

McKean, H.P., 1970. Nagumo's equation. Adv Math 4, 209-223.

Mestl, T., Plahte, E., Omholt, S.W., 1995a. A mathematical framework for describing and analysing gene regulatory netwoks. J. Theor. Biol. 176, 291300 .

Mestl, T., Plahte, E., Omholt, S.W., 1995b. Periodic solutions in systems of piecewise-linear differential equations. Dynam. Stabil. Syst. 10, 179-193.

Mincheva, M., 2011. Oscillations in biochemical reaction networks arising from pairs of subnetworks. Bull Math Biol 73, 2277-2304.

Mincheva, M., Cracium, G., 2008. Multigraph conditions for multistability, oscillations and pattern formation in biochemical reaction networks. Proc IEEE 96, 1281-1291.

Mincheva, M., Roussel, M.R., 2007. Graph-theoretic methods for the analysis of chemical and biochemical networks. i. multistability and oscillations in ordinary differential equation models. J Math Biol 55, 61-86. 
Müller, S., Hofbauer, J., Endler, L., Flamm, C., Widder, S., Schuster, P., 2006. A generalized model of the repressilator. J Math Biol 53, 905-937.

Pigolotti, S., Krishna, S., Jensen, M.H., 2007. Oscillation patterns in negative feedback loops. Proc. Natl. Acad. Sci. USA 104, 6533-6537.

Plahte, E., Kjoglum, S., 2005. Analysis and generic properties of gene regulatory networks with graded response functions. Physica D 201, 150-176.

Plahte, E., Mestl, T., Omholt, W.S., 1995. Feedback loops, stability and multistationarity in dynamical systems. J. Biol. Sys. 3, 409-413.

Purcell, O., Savery, N.J., Grierson, C.S., di Bernardo, M., 2010. A comparative analysis of synthetic genetic oscillators. J. R. Soc. Interface 7, 1503-1524.

Richard, A., Comet, J.P., 2011. Stable periodicity and negative circuits in differential systems. J. Math. Biol. 63, 593-600.

Smith, H., 1987. Oscillations and multiple steady states in a cyclic gene model with repression. J. Math. Biol. 25, 169-190.

Smith, H.L., 1986. Periodic orbits of competitive and cooperative systems. J. Diff. Equations 65, 361-373.

Snoussi, E.H., 1989. Necessary conditions for multistationarity and stable periodicity. Dyn. Stab. Syst. 4, 189-207.

Snoussi, E.H., 1998. Qualitative dynamics of piecewise-linear differential equations. J Biol Syst 6, 3-9.

Snoussi, E.H., Thomas, R., 1993. Logical identification of all steady states: the concept of feedback loop characteristic states. Bull Math Biol 55, 973-991.

Strelkowa, N., Barahona, M., 2010. Switchable genetic oscillator operating in quasi-stable mode. J. R. Soc. Interface 7, 1071-1082.

Stricker, J., Cookson, S., Bennett, M.R., Mather, W.H., Tsimring, L.S., Hasty, J., 2008. A fast, robust and tunable synthetic gene oscillator. Nature 456, $516-519$.

Thomas, R., 1981. On the relation between the logical structure of systems and their ability to generate multiple steady states or sustained oscillations. Springer Ser. Synergetics 9, 180-193.

Tsai, T.Y..C., Choi, Y.S., Ma, W., Pomerening, J.R., Tang, C., Ferrell, J.E.J., 2008. Robust, tunable biological oscillations from interlinked positive and negative feedback loops. Science 321, 126-129.

Tyson, J.J., 1975. On the existence of oscillatory solutions in negative feedback cellular control processes. J Math Biol 1, 311-315. 
Walker, J.J., Spiga, F., Waite, E., Zhao, Z., Kershaw, Y., Terry, J.R., Lightman, S.L., 2012. The origin of glucocorticoid hormone oscillations. PLoS Biol 10. doi:10.1371/journal.pbio.1001341.

Weber, A., Sturm, T., Abdel-Rahman, E.O., 2011. Algorithmic global criteria for excluding oscillations. Bull Math Biol 73, 899-916. 